\title{
Current landscape and future of dual anti- CTLA4 and PD-1/PD-L1 blockade immunotherapy in cancer; lessons learned from clinical trials with melanoma and non- small cell lung cancer (NSCLC)
}

\author{
Young Kwang Chae ${ }^{1,2,3^{*}}$, Ayush Arya ${ }^{1}$, Wade lams ${ }^{3}$, Marcelo R. Cruz ${ }^{1}$, Sunandana Chandra ${ }^{1,2,3}$, Jaehyuk Choi ${ }^{2,3}$ \\ and Francis Giles ${ }^{1,2,3}$
}

\begin{abstract}
Immunotherapy is among the most rapidly evolving treatment strategies in oncology. The therapeutic potential of immune-checkpoint inhibitors is exemplified by the recent hail of Food and Drug Administration (FDA) approvals for their use in various malignancies. Continued efforts to enhance outcomes with immunotherapy agents have led to the formulation of advanced treatment strategies. Recent evidence from pre-clinical studies evaluating immune-checkpoint inhibitors in various cancer cell-lines has suggested that combinatorial approaches may have superior survival outcomes compared to single-agent immunotherapy regimens. Preliminary trials assessing combination therapy with anti-PD-1/ PD-L1 plus anti-CTLA-4 immune-checkpoint inhibitors have documented considerable advantages in survival indices over single-agent immunotherapy. The therapeutic potential of combinatorial approaches is highlighted by the recent FDA approval of nivolumab plus ipilimumab for patients with advanced melanoma. Presently, dual-immune checkpoint inhibition with anti-programmed death receptor-1/programmed cell death receptor- ligand-1 (anti-PD-1/PD-L1) plus anti-cytotoxic T lymphocyte associated antigen-4 (anti-CTLA-4) monoclonal antibodies (MoAbs) is being evaluated for a wide range of tumor histologies. Furthermore, several ongoing clinical trials are investigating combination checkpoint inhibition in association with traditional treatment modalities such as chemotherapy, surgery, and radiation. In this review, we summarize the current landscape of combination therapy with anti-PD-1/PD-L1 plus anti-CTLA-4 MoAbs for patients with melanoma and non-small cell lung cancer (NSCLC). We present a synopsis of the prospects for expanding the indications of dual immune-checkpoint inhibition therapy to a more diverse set of tumor histologies.
\end{abstract}

\section{Background}

The regulation of immune responses through MoAbs is a ground-breaking therapeutic strategy in oncology. Based on substantial pre-clinical and clinical evidence, several immunotherapy agents have received approval by the FDA as standard of care treatment for various malignancies over the past two decades [1, 2]. However, with

\footnotetext{
* Correspondence: young.chae@northwestern.edu

'Developmental Therapeutics Program of the Division of Hematology Oncology, Early Phase Clinical Trials Unit, 645 N. Michigan Avenue, Suite 1006, Chicago, IL 60611, USA

${ }^{2}$ Robert H. Lurie Comprehensive Cancer Center of Northwestern University, 645 N. Michigan Avenue, Suite 1006, Chicago, IL 60611, USA Full list of author information is available at the end of the article
}

increasing experience in the use of immunotherapy agents in clinical settings, several limitations, such as treatment resistance and undesired immunogenicity, have been observed [3, 4]. Extensive efforts have been made to meet such challenges, and novel immune checkpoints are being tested and are expected to pave the way for the next generation of immunotherapy agents [5].

The fundamental goal in advancing anti-cancer immunotherapy is to improve clinical outcomes. The use of combination checkpoint inhibition is being applied to meet this goal. This approach intends to exploit the distinct mechanisms of immunomodulation of two MoAbs in a single treatment regimen. Recent evidence suggests

(c) The Author(s). 2018 Open Access This article is distributed under the terms of the Creative Commons Attribution 4.0 International License (http://creativecommons.org/licenses/by/4.0/), which permits unrestricted use, distribution, and 
that the combined use of an anti-CTLA-4 immunecheckpoint inhibitor with an anti-PD-1/PD-L1 MoAb may have complementary action, thus yielding a higher clinical efficacy than either agent individually [6, 7]. Comprehensive data on the efficacy of MoAb combination therapy in clinical settings is warranted in order to ascertain the true therapeutic value of this treatment strategy.

Presently, combination checkpoint inhibition is being extensively evaluated for potential clinical benefit in a large number of tumor histologies. Due to positive outcomes in preliminary trials, nivolumab (IgG4 anti-PD-1 MoAb) plus ipilimumab (fully humanized IgG1 antiCTLA-4 MoAb) is one of the most enthusiastically investigated combined immunotherapy regimens, with over 100 clinical trials in various stages $[8,9]$. Of note, nivolumab plus ipilimumab received approval for use in BRAF V600 wild-type metastatic/unresectable melanoma, making it the first combination checkpoint inhibition regimen to be approved by the FDA [9]. In addition, other PD-1/PD-L1 inhibitors plus CTLA-4 inhibitor combination checkpoint inhibition regimens that are presently in clinical trials include atezolizumab (anti-PD-L1 MoAb) plus ipilimumab, pembrolizumab (IgG4 anti-PD-1 MoAb) plus ipilimumab, and tremelimumab (IgG2 anti-CTLA-4 MoAb) plus durvalumab (Fc optimized anti-PD-L1 MoAb) [10]. The data published from these trials will be crucial to appraise the efficacy of combination immune checkpoint inhibitor regimens in varying clinical scenarios.
In this review, we describe the rationale for combined immunotherapy with PD-1/PD-L1 plus CTLA-4 checkpoint inhibitors. Building on what we have learned through studies of combination checkpoint inhibition in patients with melanoma and NSCLC, we shall also critically assess the current landscape and future prospects for the development of an ideal combination checkpoint inhibition regimen.

\section{Role of PD-1/PD-L1 and CTLA-4 in modulation of anti- tumor T-cell activity}

The process of $\mathrm{T}$ cell activation requires two signals. The primary signal comes from the binding of the T cell receptor (TCR) to the major histocompatibility complex (MHC) molecule presented by an antigen presenting cell (APC) [11]. The costimulatory signal may arise from one of several distinct T cell-APC interactions. One such pathway is the engagement of CD28 on T cells with CD80 (B7-1) or CD86 (B7-2) on APCs [11] (Fig. 1). T-cell activity can be modulated by regulating the generation of costimulatory signals through various mechanisms.

Several signaling pathways have been implicated in the modulation of $\mathrm{T}$ cell activity. The CTLA- 4 molecule is a homolog of CD28 and is expressed by T cells (Fig. 1) $[12,13]$. The influence of CTLA-4 on T cell activity primarily occurs in the priming phase of $\mathrm{T}$ cell activation [14]. CTLA-4 competitively binds to B7 on APCs and inhibits the costimulatory signal that arises from the engagement of $\mathrm{CD} 28$ with $\mathrm{B} 7$, thereby diminishing the $\mathrm{T}$ cell immune response $[13,15-18]$. The upregulation of

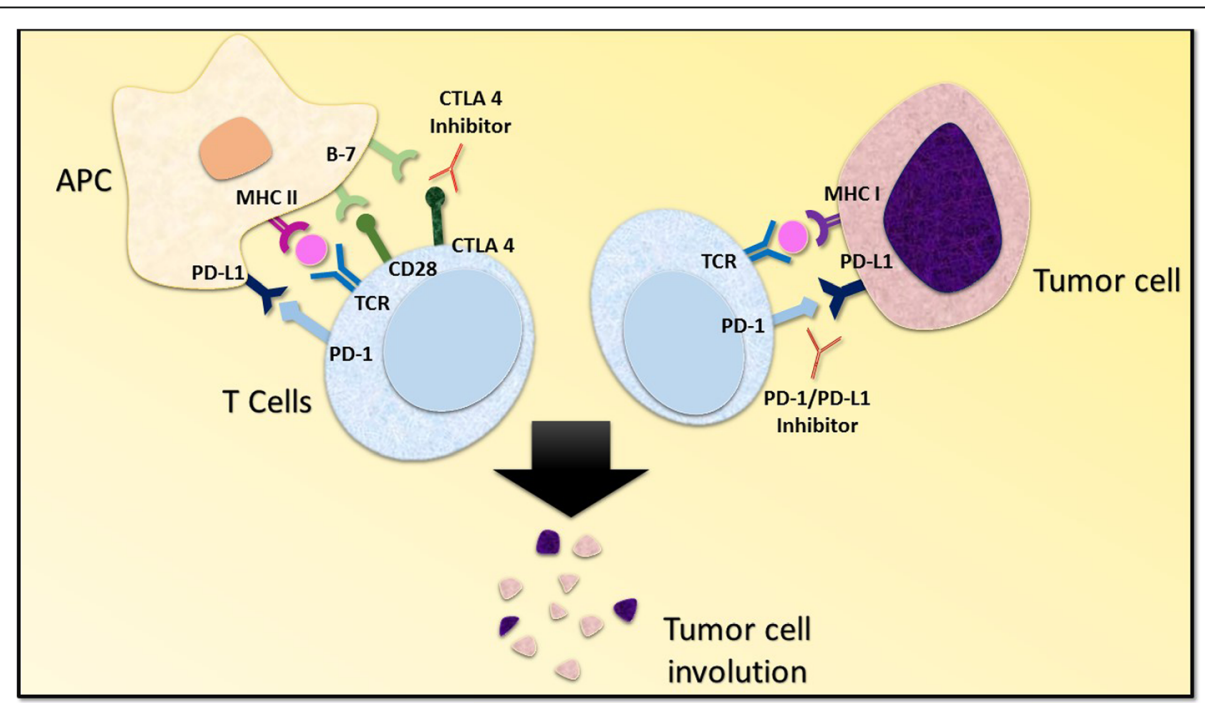

Fig. 1 Mechanism of CTLA 4 and PD-1/PD-L1 inhibition. The activation of T cells is mediated by the interaction of T cell receptor and the CD28 receptor with class II major histocompatibility complex and B7 co-stimulatory molecule located on the antigen presenting cells. The interaction of CTLA-4 with the B7 molecule delivers an inhibitory signal, effectively checked by CTLA-4 inhibitors. On the other hand, the negative regulation of T cells resulting from PD-1/PD-L1 interaction between T cells and tumor cells is prevented by PD-1/PD-L1 inhibitors. Abbreviations: APC, antigen presenting cell; PD-1, programmed death receptor-1; PD-L1, programmed cell death receptor ligand-1; TCR, T cell receptor; MHC I, major histocompatibility complex class I; MHC II, major histocompatibility complex class II 
CTLA-4 expression on CD8+ and CD4+ T cells precludes stimulatory signaling from CD28-B7 binding and TCR-MHC binding $[11,19]$. On the other hand, regulatory $\mathrm{T}$ cells $\left(\mathrm{T}_{\text {reg }}\right.$ cells) exhibit constitutive expression of CTLA-4 [20]. Murine models bearing $\mathrm{T}_{\text {reg }}$ cells deficient in CTLA-4 exhibited attenuated immunosuppressive activity, thus highlighting the significance of CTLA-4 in regulating immunological self-tolerance [20].

The PD-1 molecule is akin to CTLA-4. It is a member of the B7-CD28 family and is expressed by myeloid derived cells, B cells, and T cells [21, 22]. PD-1 has two corresponding ligands, PD-L1 and PD-L2 [14]. PD-L1 is expressed by a diverse set of cells including hematopoietic cells, leucocytes, parenchymal cells, and tumor cells, whereas PD-L2 is expressed by dendritic cells and macrophages [22-24]. The PD-1 receptor on T cells binds PDL1 expressed by APCs and inhibits pro-inflammatory events such as $\mathrm{T}$ cell proliferation and cytokine production (Fig. 1) [14, 25]. Of note, recent evidence has suggested that PD-1/PD-L1 interactions facilitate immune escape by tumor cells $[24,26]$. This phenomenon has been attributed to PD-1/PD-L1 mediated induction of anergy and apoptosis of activated T cells, tumor resistance to the cytotoxic $\mathrm{T}$ cell response, and differentiation of $\mathrm{CD} 4+\mathrm{T}$ cells into Fox3p + CD4+ $\mathrm{T}_{\text {reg }}$ cells [27-29].

An intricate knowledge of various pathways regulating $\mathrm{T}$ cell-APC interactions has been central to identifying the points of intervention that allow us to modulate host immune responses. The aforementioned evidence and other studies with similar outcomes prompted the development of PD-1/PD-L1 and CTLA-4 checkpoint inhibitors for potential application in anti-cancer therapy.

\section{Preclinical rationale for combination checkpoint inhibition} Combination immunotherapy with PD-1/PD-L1 plus CTLA-4 checkpoint inhibitors has been studied in multiple cancer cell lines. In an experiment on murine preclinical models, vaccination with B16-Flt-3 ligand (Fvax) along with the use of CTLA-4 antibody promoted tumor rejection in $10 \%$ of mice with pre-implanted B16-BL6 melanoma [7]. Fvax plus PD-1 blockade exhibited tumor rejection in $25 \%$ of mice. The combined use of CTLA-4 and PD-1 checkpoint inhibitors resulted in the rejection of B16-BL6 melanoma in 50\% of test animals. Upon the addition of a PD-L1 inhibitor to the above, $65 \%$ of test animals exhibited rejection of melanoma. The outcomes observed with combined PD-1 and CTLA- 4 blockade were found to correlate with an increase in CD4+ effector T cell $\left(\mathrm{CD} 4+\mathrm{T}_{\text {eff }}\right)$ to $\mathrm{T}_{\text {reg }}$ cell ratio and CD8+ $\mathrm{T}$ cell to $\mathrm{T}_{\text {reg }}$ cell ratio in tumor tissue. Another significant observation was that a high percentage of T cells positive for CTLA- 4 and PD-1 that would have undergone anergy remained active with combined PD-1 plus CTLA-4 blockade [7].
Similar findings were documented in another study investigating the effects of checkpoint inhibition (PD-1/PDL1 and CTLA-4) in murine models of ovarian (ID8-VEGF) and colonic carcinoma (CT26) [30]. For ID8-VEGF, tumor regression was observed in 25\% of test animals after PD-1 blockade, 25\% with CTLA-4 blockade, and 37.5\% with PD-L1 blockade, as compared to 50\% with combined CTLA-4 plus PD-1 or PD-L1 blockade. Likewise, in mice bearing CT26, tumor regression was observed in $25 \%$ of test animals with PD-1 blockade, $50 \%$ with CTLA-4 blockade, and 33\% with PD-L1 blockade, as compared to 75\% $(p<0.01$ for the combination to checkpoint inhibitor monotherapy comparison) with combined CTLA-4 plus PD-1 or PD-L1 blockade. Combined therapy with CTLA-4 and PD-1 blockade was associated with a greater enhancement in tumor induced lymphocyte (TIL) activity and proliferation similar to CTLA-4 or PD-1 blockade alone [30]. Combination therapy also decreased the frequency of $\mathrm{T}_{\mathrm{reg}}$ cells and functional markers of activated $\mathrm{T}_{\text {reg }}$ cells such as glucocorticoid induced tumor necrosis factor receptor (GITR) [30]. This suggested that dual checkpoint inhibition simultaneously blunted the function and decreased the number of $\mathrm{T}_{\text {reg }}$ cells.

In view of these and other preclinical studies, it was established that combined immune checkpoint blockade decreases suppression of the host immune system, while promoting inflammation in the tumor microenvironment. Moreover, the vast amount of preclinical data also suggested that the anti-tumor activity of combination therapy with CTLA-4 plus PD-1/PD-L1 checkpoint inhibitors may have superior outcomes compared to CTLA-4 or PD-1 monotherapy. It was thus prudent to investigate the therapeutic potential of CTLA-4 plus PD-1/PD-L1 checkpoint inhibitors in clinical settings.

\section{Emergence of combination checkpoint inhibition}

Cancer immunosurveillance has been considered one of the primary natural mechanisms of defense against aberrant cell populations [31, 32]. Immune cells recognize and eliminate transformed cells through various cellular interactions [33]. In addition, the immune system is also involved in shaping tumor immunogenicity, a phenomenon theorized as immunoediting [34]. However, it was observed that cancer cells gradually undergo immune selection that disrupts the equilibrium with immune cells, consequently generating a tumor cell population that effectively evades immune surveillance [35-38]. Furthermore, alterations in the tumor microenvironment have also been reported to mediate immune escape [39]. It was thus theorized that therapeutic agents with the ability to restore immune surveillance or prevent immune escape of tumor cell populations could potentially have a significant impact in clinical oncology.

The knowledge of various factors that influence tumorigenesis has been exploited for developing a wide 
range of immunotherapy agents. Immunogenic cell death inducers, immunostimulatory cytokines, pattern recognition receptor agonists (PRR agonists), and tumor targeting antibodies have benefitted cancer patients for nearly two decades [40]. Numerous immunotherapy agents have received FDA approval for use as monotherapy in a variety of tumor histologies. For instance, nivolumab has been approved for patients with melanoma, renal cell carcinoma (RCC), metastatic squamous cell carcinoma of the head and neck (SCCHN), classical Hodgkin's lymphoma (HL) and advanced lung cancer [41-46]. Pembrolizumab, another anti-PD-1 MoAb, is approved for use in patients with SCCHN, NSCLC, and melanoma [47-49]. Likewise, atezolizumab is approved for patients with NSCLC and urothelial carcinoma [50-52]. However, any further enhancements in clinical benefit beyond checkpoint inhibitor monotherapy are still in clinical trials.

Recent clinical trial data comparing combination therapy with nivolumab and ipilimumab versus ipilimumab monotherapy in treatment naive melanoma patients drew much attention (CheckMate 069, NCT01927419). In patients with $B R A F$-wild type melanoma, the investigators reported an objective response of $61 \%$ (95\% CI: 49-72) as opposed to $11 \%$ (95\% CI: 3-25) with combination checkpoint inhibition and ipilimumab monotherapy, respectively [53]. Furthermore, $22 \%$ of study participants receiving combination therapy exhibited a complete response, as compared to none among those receiving ipilimumab monotherapy [53]. In view of these findings, combined therapy with nivolumab and ipilimumab in melanoma patients became the first FDA approved (accelerated approval) indication for combination checkpoint inhibition. Furthermore, this study prompted comprehensive efforts to explore the application of combined immunotherapy with anti-PD-1/PDL1 and anti-CTLA-4 MoAbs for patients with various malignancies.

Currently there are a large number of clinical trials evaluating several PD-1/PD-L1 plus CTLA-4 checkpoint inhibitor combination regimens. Positive outcomes in preliminary trials have made way for more intensive efforts to explore the full potential of combination checkpoint inhibition regimens, particularly nivolumab plus ipilimumab. It is widely anticipated that the ongoing trials will provide formidable evidence to support clinical applications of PD-1/PD-L1 plus CTLA-4 checkpoint inhibitor combination regimens for patients with a substantial variety of tumor histologies.

\section{Methods}

The details of pertinent clinical trials were gathered from clinicaltrials.gov (United States National Institute of Health) from the database available to public domain. The database was narrowed using the search queries "nivolumab" with "ipilimumab", "pembrolizumab" with "ipilimumab", "atezolizumab" with "ipilimumab" and "tremelimumab" with "durvalumab". All prospective clinical trials using aforementioned immunotherapy agents as treatment intervention in NSCLC or melanoma patients were selected from the filtered results. Additionally, all phase 1 solid tumor trials aimed at providing a recommendation for the appropriate phase 2 dose of above combinations were also selected. The NCT number of each trial was used to search for published results on online databases including PubMed, American Association of Cancer Research (AACR), American Society of Clinical Oncology (ASCO) and European Society for Medical Oncology (ESMO).

\section{Clinical trials: Current landscape}

The United States National Institutes of Health lists a total of 44 ongoing clinical trials (clinicaltrials.gov) evaluating combined immunotherapy with anti-PD-1/ PD-L1 plus anti-CTLA-4 MoAbs for patients with NSCLC and melanoma (Tables 1 and 2, Fig. 2a). The designated combination therapy regimens include nivolumab plus ipilimumab, pembrolizumab plus ipilimumab, atezolizumab plus ipilimumab, and tremelimumab plus durvalumab. A majority of these trials focus on survival and other treatment response indices, whereas a limited number of trials are investigating the safety profile and maximum tolerable dose (MTD) of combination therapy protocols. Furthermore, eight phase 1/phase 2 clinical trials are in the process of enrolling participants with solid tumors in order to determine recommended phase 2 doses (RP2D) for combination checkpoint inhibitor therapy with various anti-PD-1/PD-L1 and antiCTLA-4 MoAbs. Of note, over three quarters (37 of 44 trials) of ongoing combination therapy trials for patients with NSCLC and melanoma are investigating treatment regimens that involve the application of nivolumab and ipilimumab (Fig. 2a and b).

\section{Combination checkpoint inhibition trials in melanoma}

Massive efforts are testing dual checkpoint inhibition with anti-PD-1/PD-L1 plus anti-CTLA-4 MoAbs in patients with melanoma. Presently, there are 28 clinical trials in various phases committed to this objective (Table 1). Currently, only one trial is in the process of recruiting participants that will receive ipilimumab in combination with pembrolizumab. Approximately twothirds of all ongoing trials (18 of 28 trials) in melanoma patients are investigating combined therapy with nivolumab plus ipilimumab.

Several distinct methodologies for the combined use of nivolumab and ipilimumab have been identified and are presently being investigated for potential clinical benefit in two trials. One trial will compare the incidence of adverse events, overall response rate (ORR), 


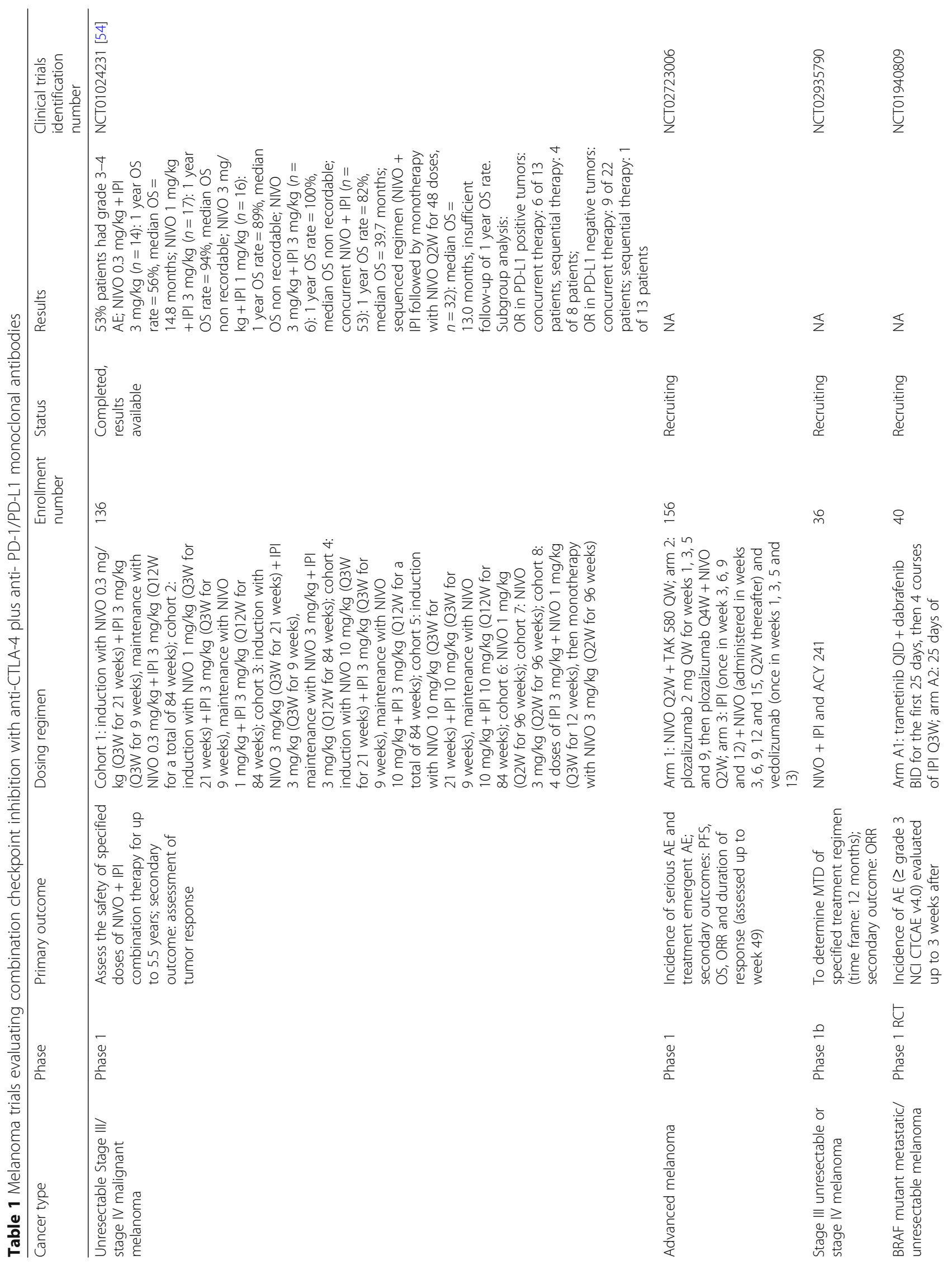




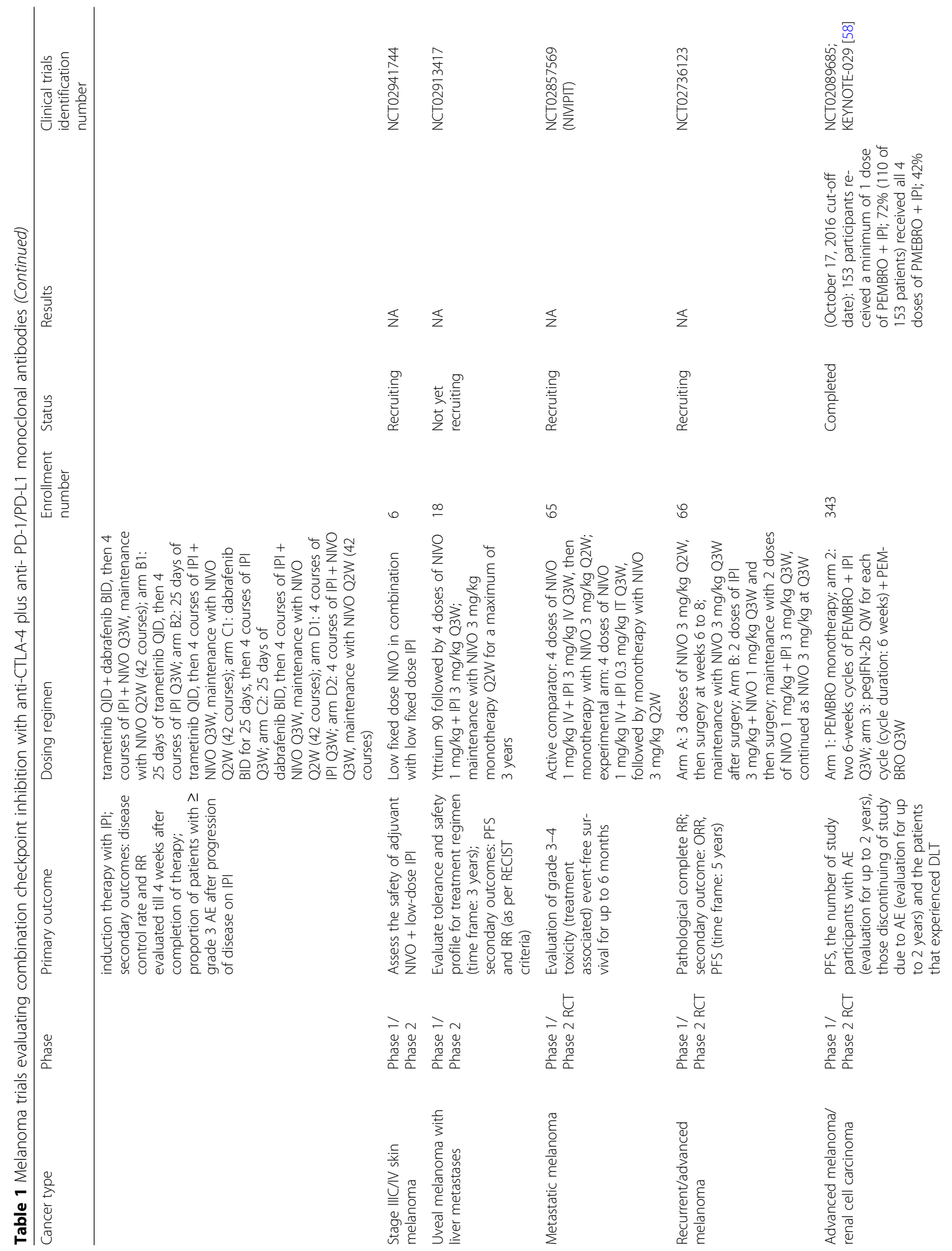




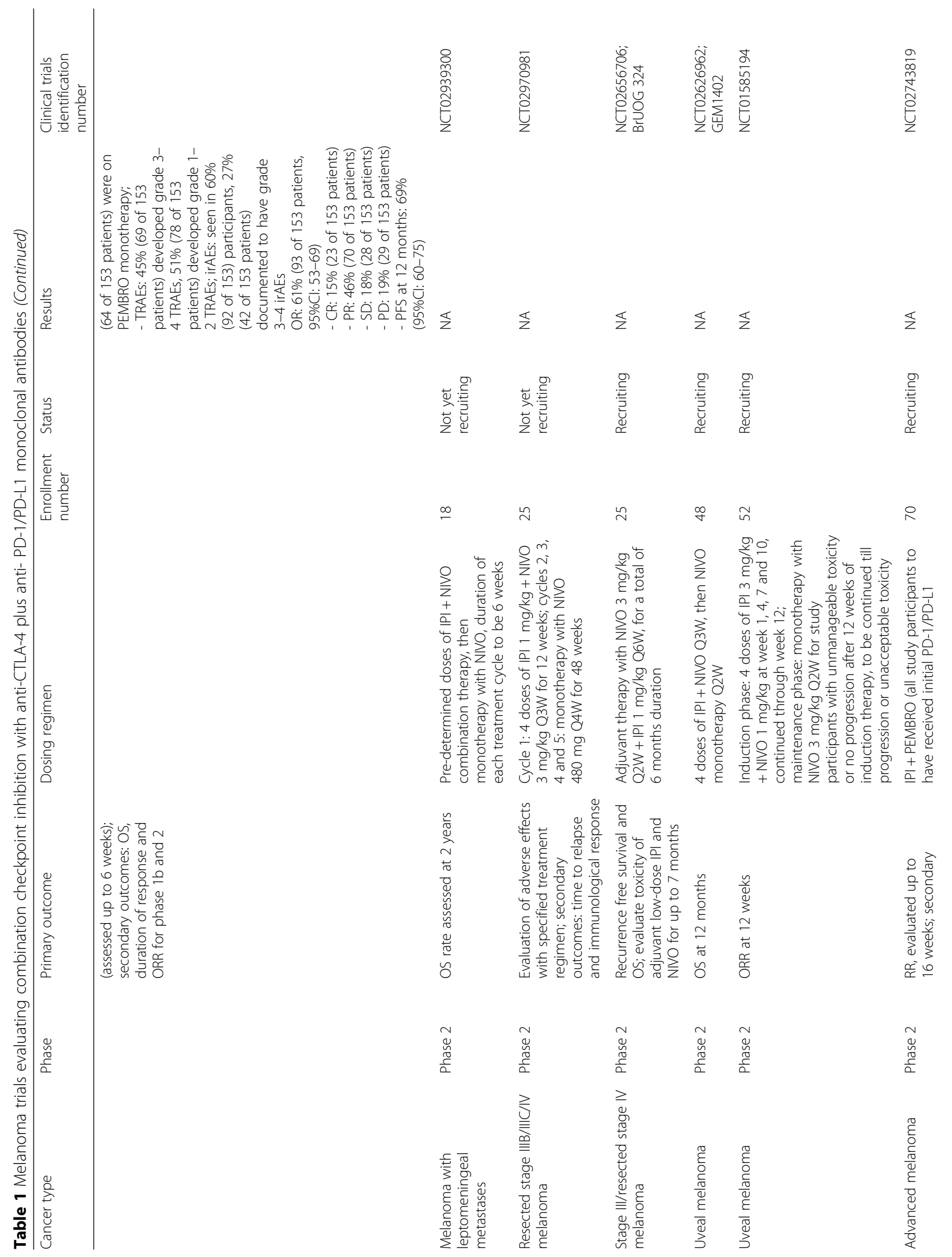




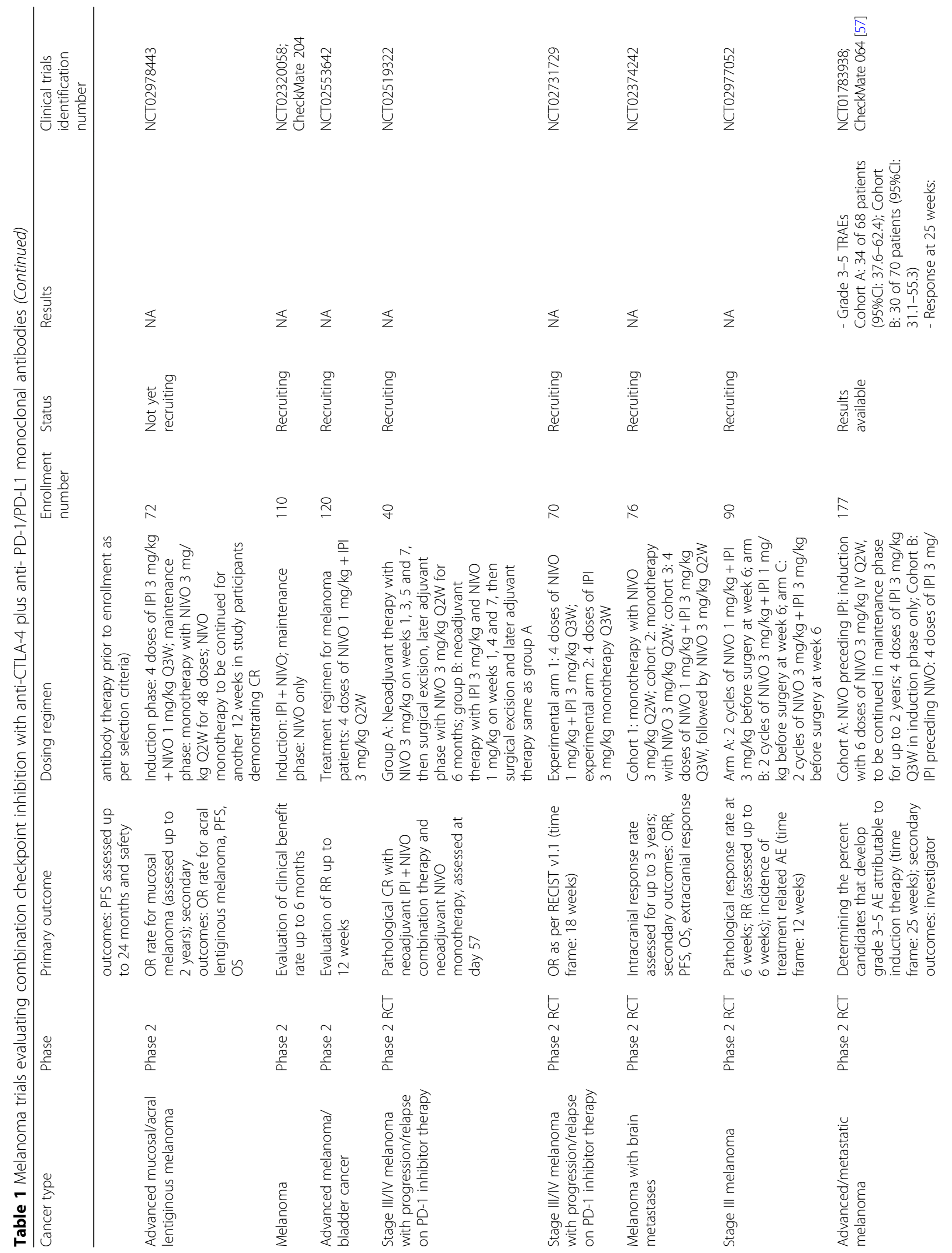




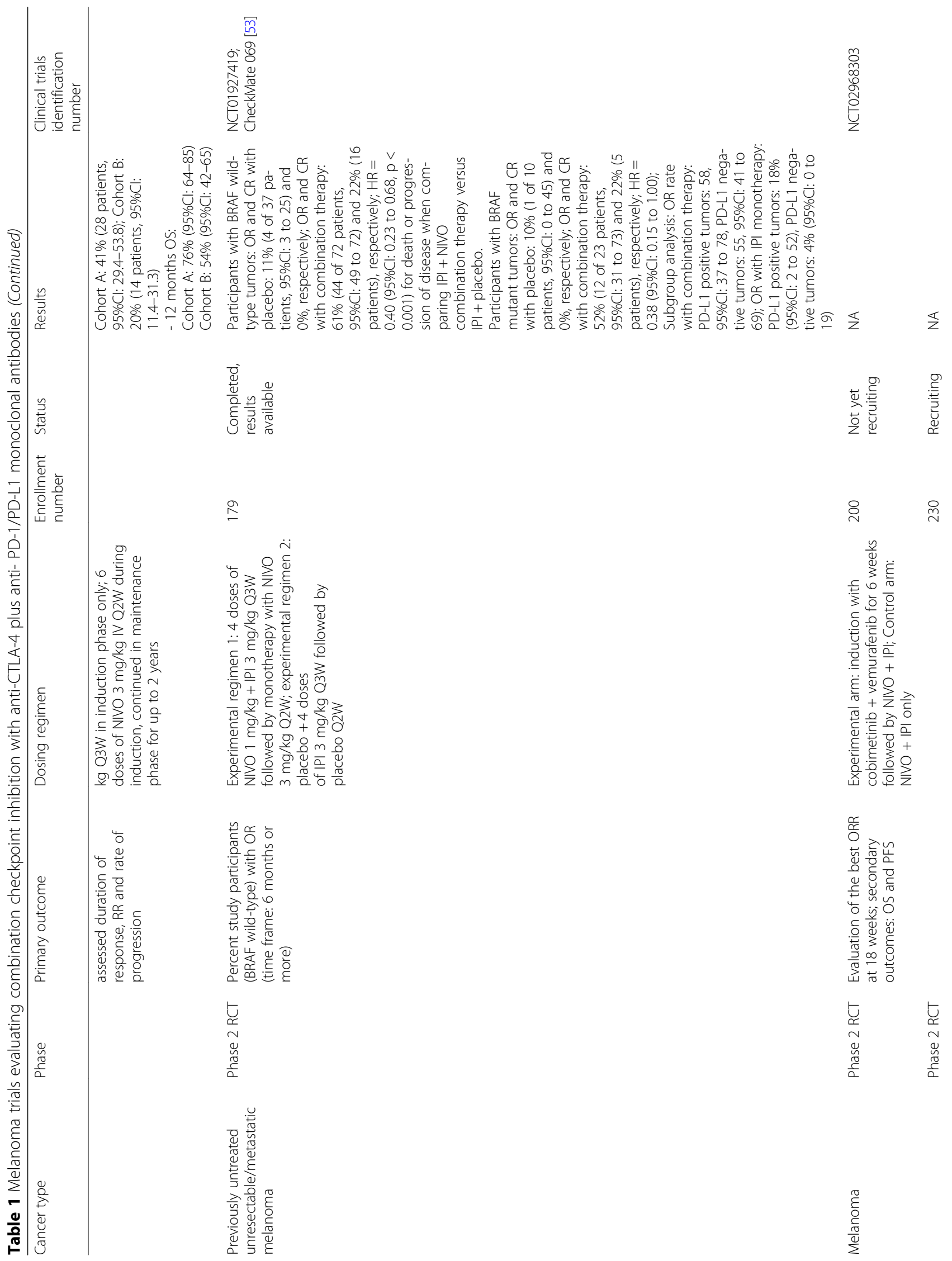




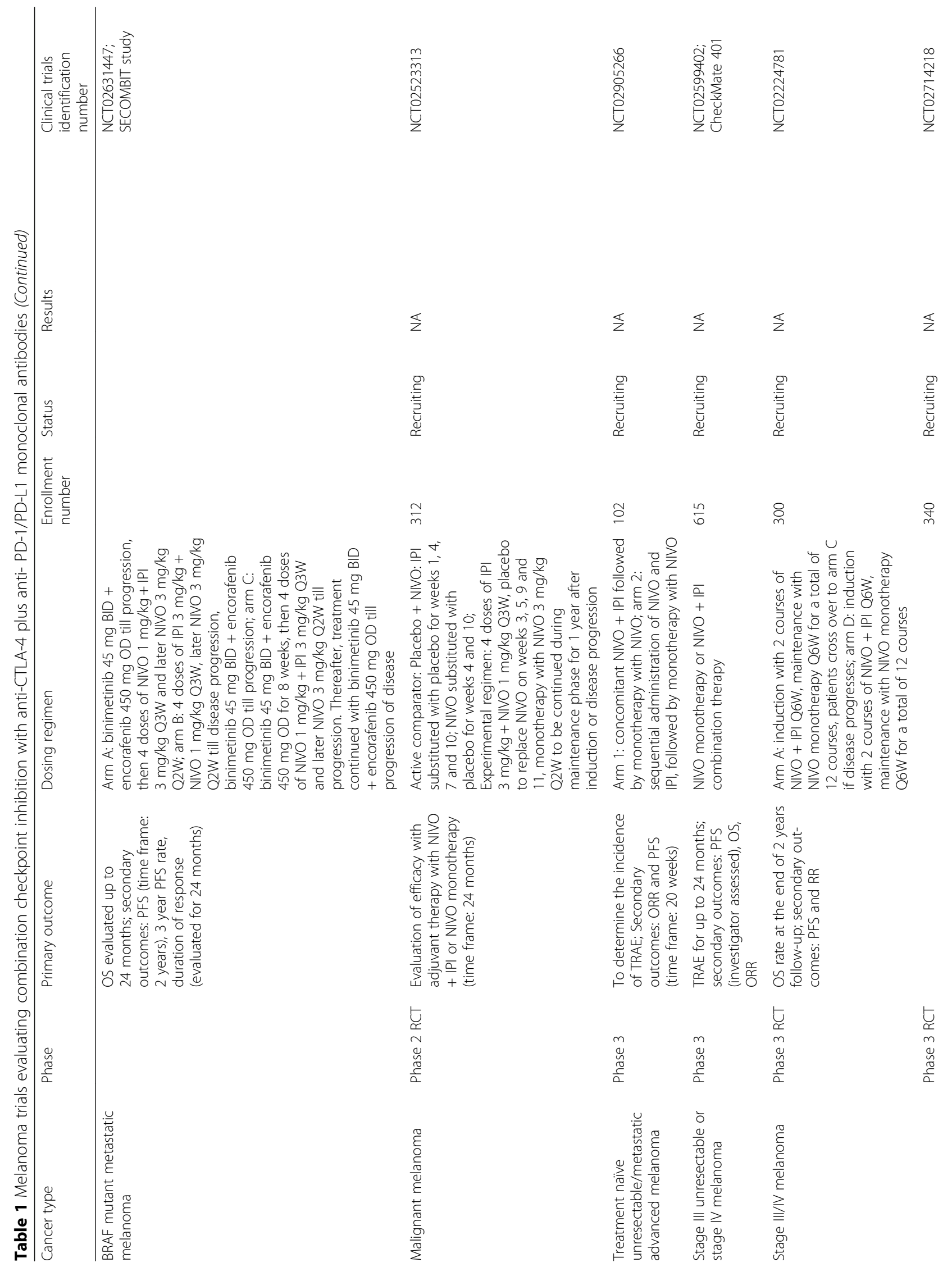




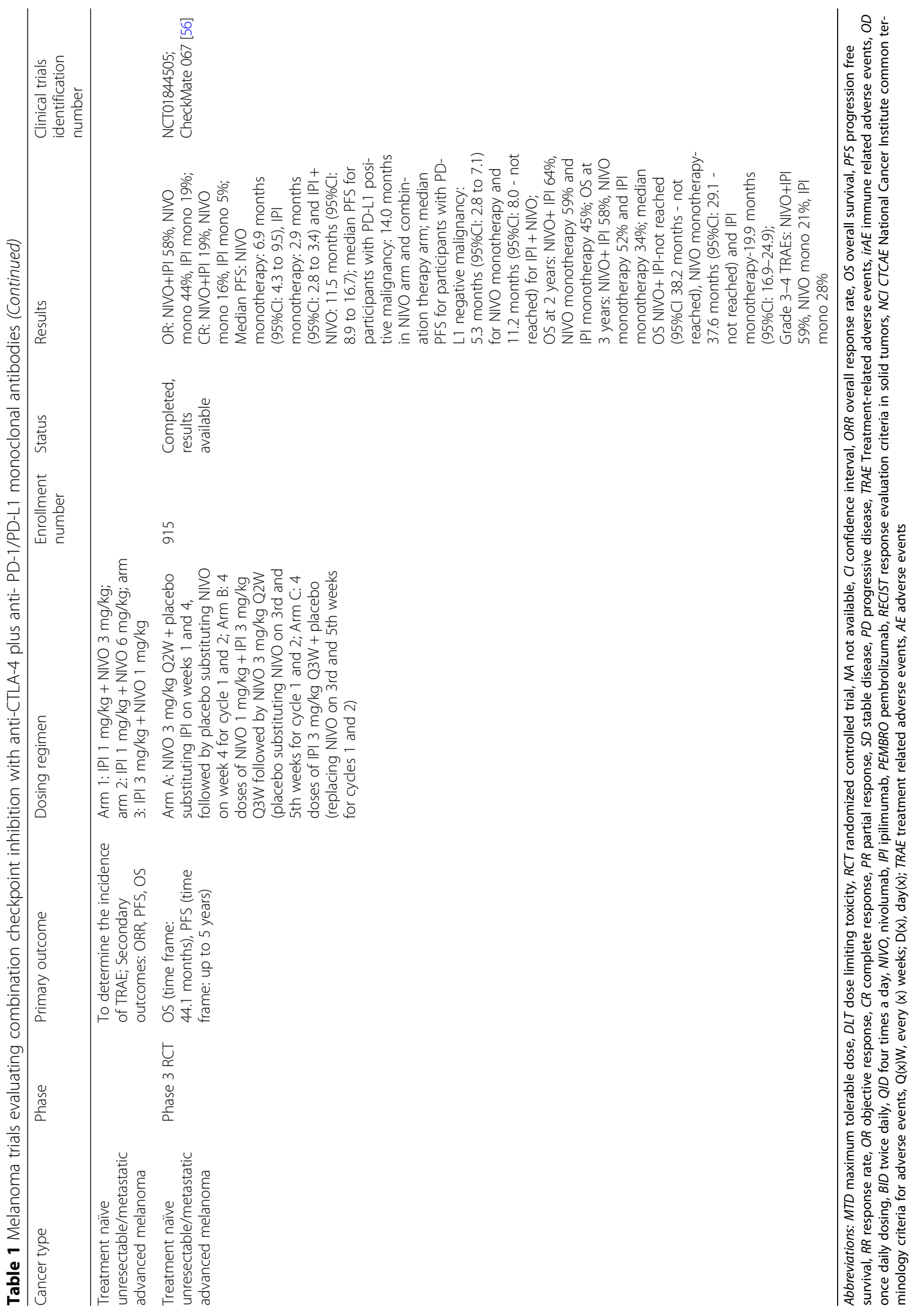

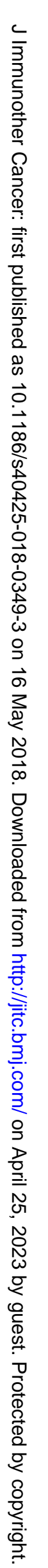




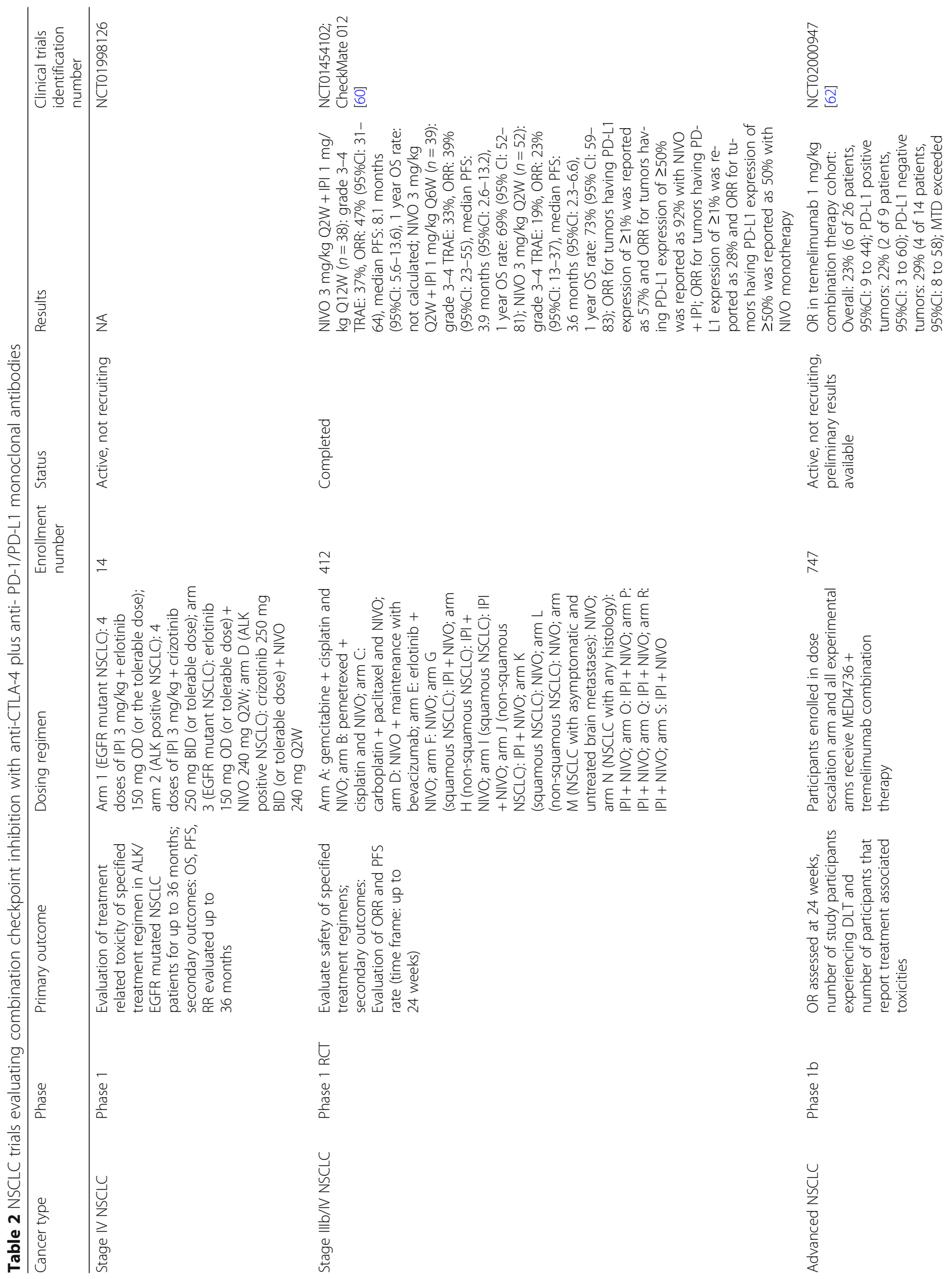

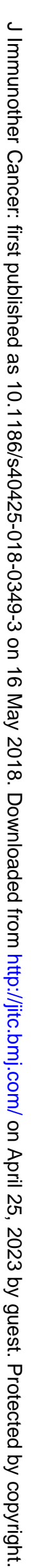




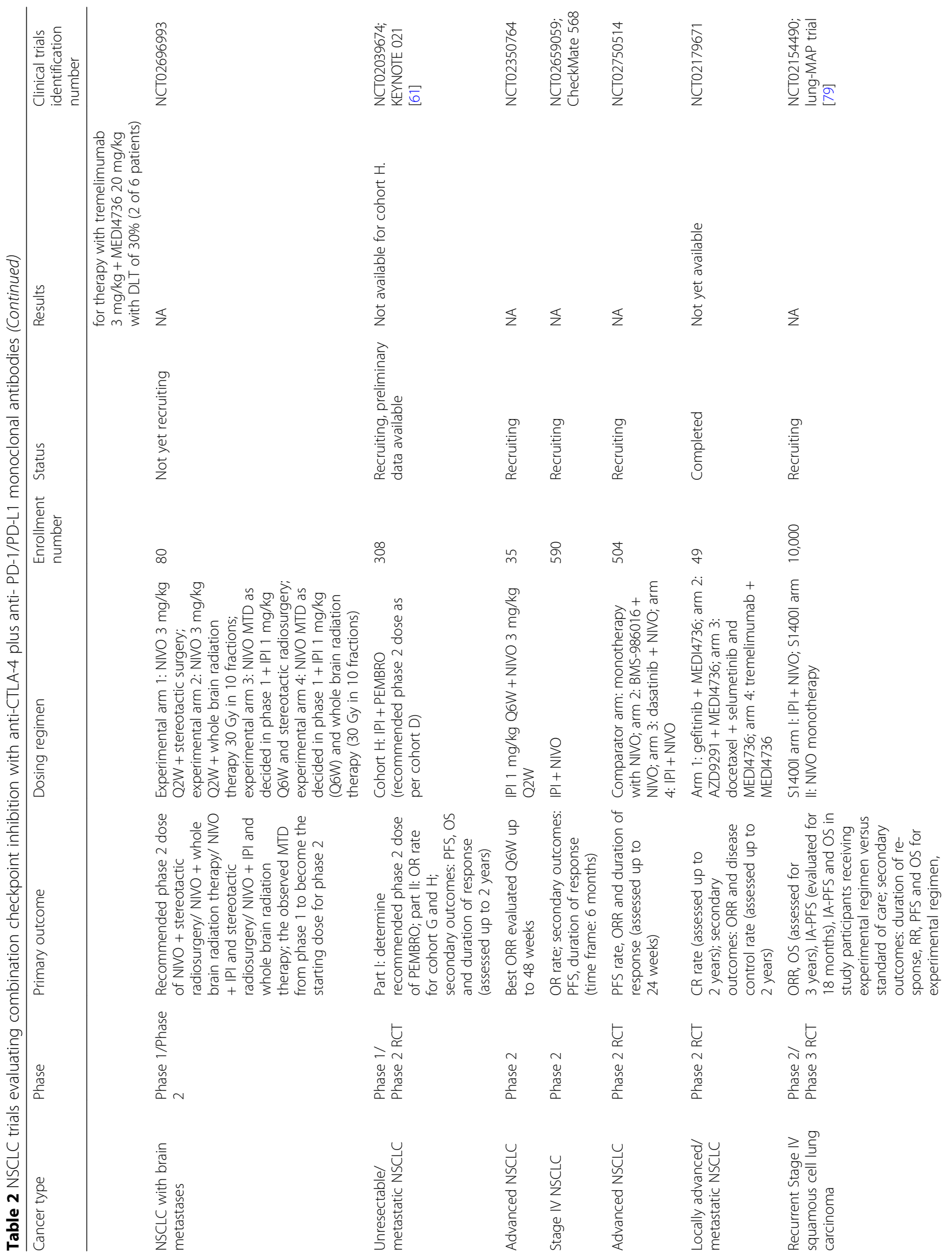

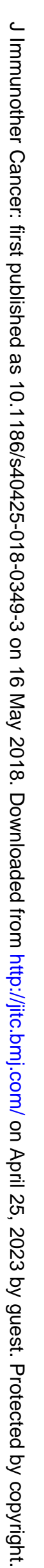




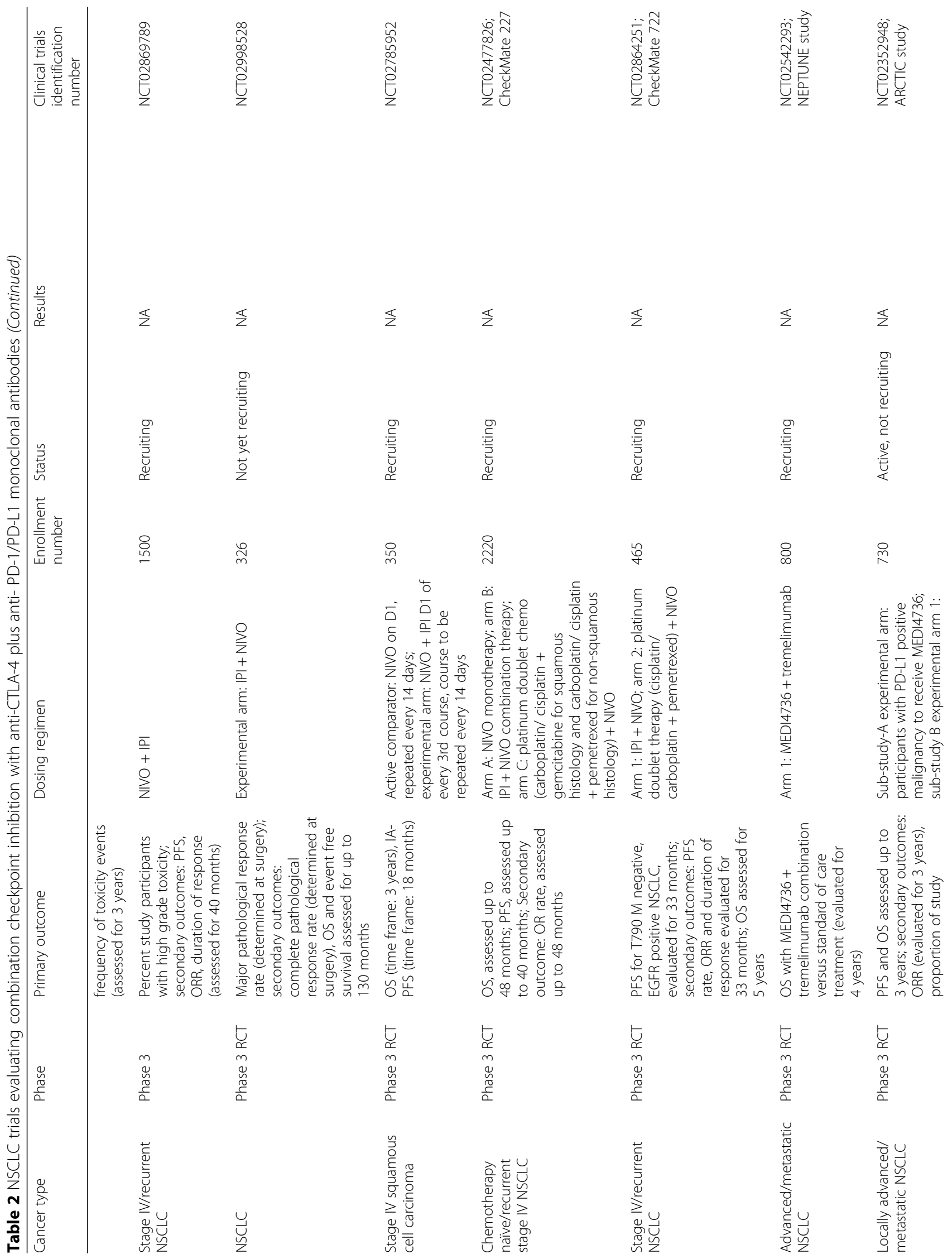




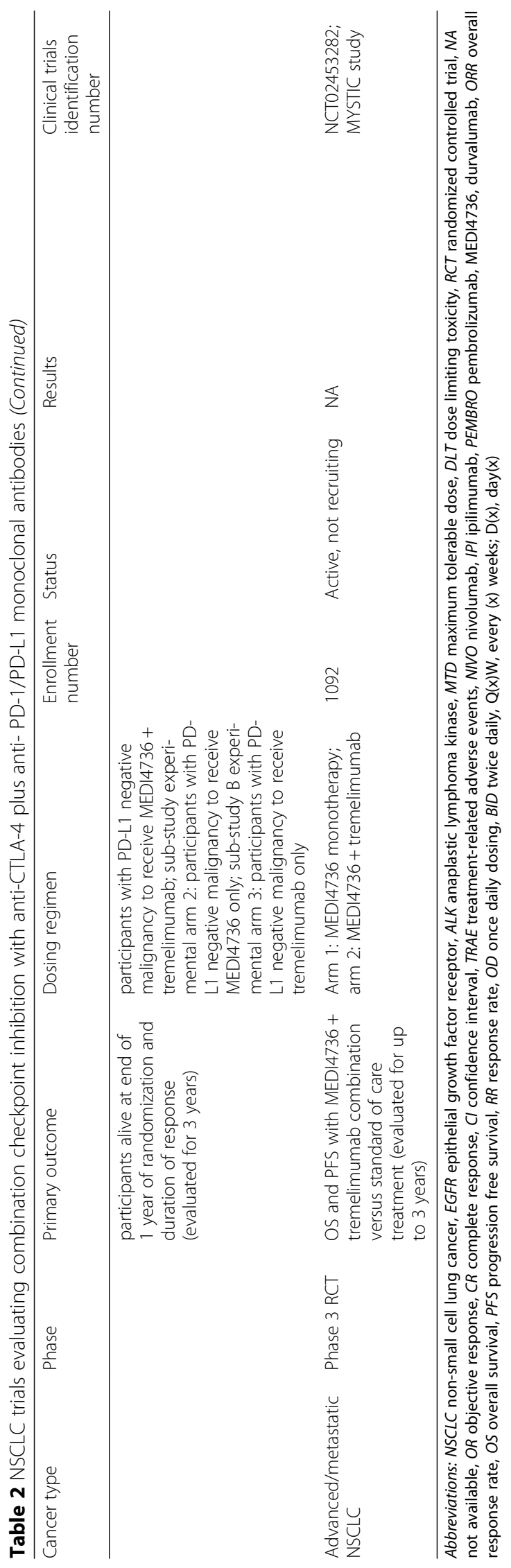

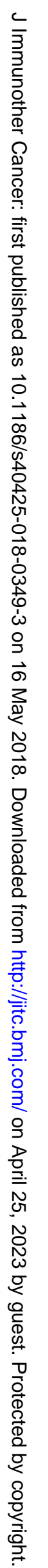



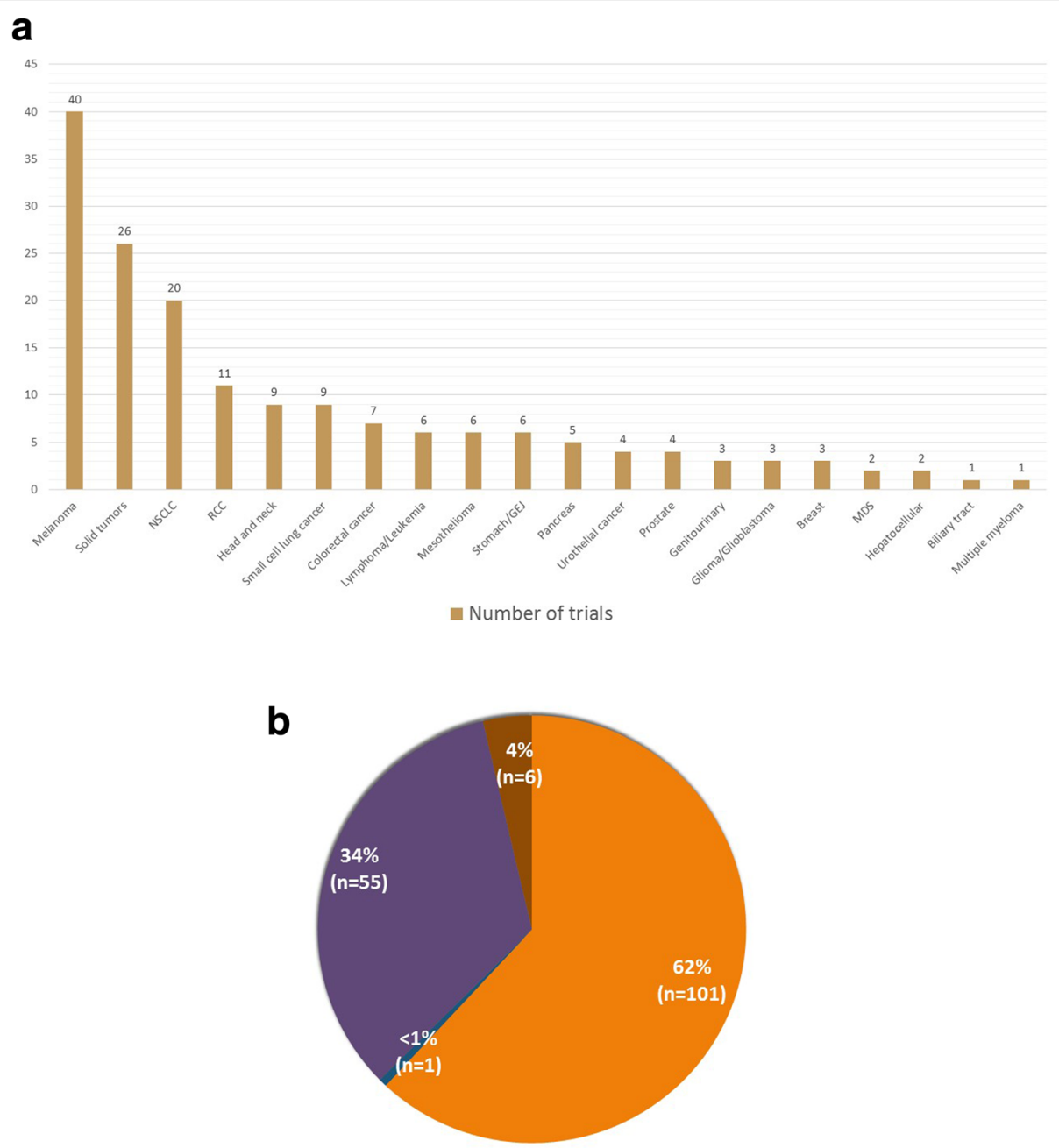

\footnotetext{
Nivolumab + Ipilimumab Atezolizumab + Ipilimumab

- Tremelimumab + Durvalumab $\mathbf{n}$ Pembrolizumab + Ipilimumab
}

Fig. 2 Current landscape of combination immunotherapy trials for various tumor histologies. a Number of combination checkpoint inhibition trials for various tumor histologies. Abbreviations: NSCLC, non-small cell lung carcinoma; RCC, renal cell carcinoma; GEJ, gastro-esophageal junction; MDS, myelodysplastic syndrome. $\mathbf{b}$ Landscape of combination checkpoint inhibition agents. Legend: The figure elaborates relative number of trials for four combinations of immunotherapy agents. Nivolumab plus ipilimumab: 62\% (101 trials), pembrolizumab plus ipilimumab $4 \%$ (6 trials), tremelimumab plus durvalumab 34\% (55 trials) and atezolizumab plus ipilimumab $<1 \%$ (1 trial)

and progression free survival (PFS) with concurrent versus sequential administration of nivolumab and ipilimumab in the induction phase (NCT02905266). Another trial, CheckMate 064, will evaluate the duration of response, response rate (RR), rate of progression and the proportion of study participants that develop grade $3-5$ adverse events when using nivolumab prior to ipilimumab versus ipilimumab prior to nivolumab in the induction phase (NCT01783938).

Treatment protocols in five trials involve the integrated use of nivolumab and/or ipilimumab with additional anticancer agents. The assessment of MTD and ORR for combined therapy with nivolumab plus ipilimumab and ACY241 (histone deacetylase (HDAC $\}$ inhibitor) will be performed in one trial (NCT02935790) [10]. Another trial will be exploring the clinical efficacy and incidence of adverse events for nivolumab plus TAK580 (pan-Raf kinase inhibitor), nivolumab and plozalizumab (chemokine receptor-2 (anti-CCR-2\} MoAb), and the combined use of nivolumab plus ipilimumab and vedolizumab (anti-LPAM-

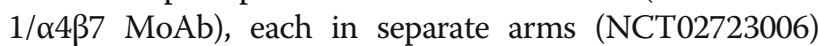
[10]. Similarly, a different trial will evaluate the safety profile and clinical efficacy with combination dabrafenib (BRAF protein inhibitor) and/or trametinib (MEK MAPK/ ERK kinase (mitogen-activated protein kinase kinase\} inhibitor) in the induction phase, followed by ipilimumab and/or nivolumab (NCT01940809) [10]. This trial will also compare the outcomes of the above treatment regimens 
with nivolumab plus ipilimumab (NCT01940809). The SECOMBIT study will compare survival outcomes among several variations of treatment regimens composed of binimetinib (MEK1/2 inhibitor), encorafenib (Raf kinase inhibitor), nivolumab and ipilimumab (NCT02631447) [10]. Lastly, one trial will analyze response to vemurafenib (BRAF-V600 kinase inhibitor) plus cobimetinib (MAP2K1/ MEK1 inhibitor) in the induction phase followed by nivolumab plus ipilimumab, versus nivolumab plus ipilimumab combination therapy alone (NCT02968303) [10].

Several additional treatment modalities with nivolumab plus ipilimumab combination therapy for melanoma is being examined in three trials. Neoadjuvant therapy with nivolumab and combined nivolumab plus ipilimumab is under evaluation in NCT02519322 and NCT02977052, respectively. A different trial will assess the use of Yttrium-90 selective internal hepatic radiation prior to induction with nivolumab plus ipilimumab (NCT02913417).

The safety and survival statistics from five clinical trials that applied combined immunotherapy with various antiPD-1/PD-L1 plus anti-CTLA-4 MoAbs has been made available. One trial enrolled 136 patients with unresectable stage III/IV malignant melanoma in eight cohorts (NCT01024231). Each cohort received a unique regimen, differing in sequence of administration and dosing for nivolumab and ipilimumab. The doses for nivolumab were 1,3 , or $10 \mathrm{mg} / \mathrm{kg}$ whereas ipilimumab was administered at 3 or $10 \mathrm{mg} / \mathrm{kg}$, per predefined criteria for each cohort. The study documented a considerable variation in OS among different cohorts. In one group, among 14 patients that received nivolumab $0.3 \mathrm{mg} / \mathrm{kg}$ plus ipilimumab $3 \mathrm{mg} /$ $\mathrm{kg}$, the 1 year OS rate was $56 \%$, whereas among six patients receiving nivolumab $3 \mathrm{mg} / \mathrm{kg}$ plus ipilimumab $3 \mathrm{mg} / \mathrm{kg}$ the 1 year OS rate was 100\% [54]. Another parameter compared in this study was median OS for concurrent and sequential administration of nivolumab and ipilimumab, which was noted to be 39.7 months and 13 . 0 months, respectively. The study also recorded objective response stratified on the basis of PD-L1 status. In patients with PD-L1 positive melanoma, an objective response was noted in 6 of 13 participants receiving concurrent therapy and 4 of 8 participants receiving sequential therapy. In the PD-L1 negative subgroup 9 of 22 patients responded with concurrent therapy and 1 of 13 patients receiving sequential therapy responded. Treatment related adverse events were noted in 93\% (grade 3-4 adverse events: $53 \%$ ) patients receiving the concurrent regimen and 73\% (grade 3-4 adverse events: 18\%) in the sequential therapy group [54].

The outcomes for combined therapy with nivolumab plus ipilimumab versus placebo plus ipilimumab in $B R A F$ wild type and $B R A F$-mutant unresectable/metastatic melanoma were recorded in one trial (CheckMate 069,
NCT01927419). Combination therapy appeared to be effective in patients with both $B R A F$-wild type (objective response: $61 \%$, 44 of 72 patients, $95 \%$ CI: 49 to 72 ; complete response: $22 \%, 16$ patients) and $B R A F$-mutant tumors (objective response: $52 \%, 12$ of 23 patients, 95\%CI: 31 to 73; complete response: $22 \%, 5$ patients) [53]. The placebo group, however, exhibited poor outcomes in both $B R A F$ wild type (objective response: $11 \%, 4$ of 37 patients, 95\%CI: 3 to 25; complete response: $0 \%$ ) and BRAF mutant tumors (objective response: $10 \%, 1$ of 10 patients, $95 \% \mathrm{CI}$ : 0 to 45 ; complete response: $0 \%$ ). A subgroup analysis in this study evaluated the influence of tumor PD-L1 status on clinical outcomes. Patients receiving combination therapy demonstrated no significant difference in objective response rates (PD-L1 positive: 58, 95\%CI: 37-78; PD-L1 negative: 55, 95\%CI: 41-69) [53].

Another trial assessing survival outcomes for combined therapy with nivolumab plus ipilimumab versus placebo in treatment naïve unresectable/metastatic melanoma reported a median PFS for nivolumab monotherapy as 6.9 months (95\%CI: 4.3 to 9.5), ipilimumab monotherapy as 2.9 months (95\%CI: 2.8 to 3.4 ), and combined therapy with ipilimumab plus nivolumab as 11.5 months (95\%CI: 8.9 to 16.7) (CheckMate 067, NCT01844505) $[55,56]$. The study reported an overall survival at 2 years for nivolumab plus ipilimumab combination as $64 \%$, nivolumab monotherapy as $59 \%$ and ipilimumab monotherapy as $45 \%$. At 3 years follow-up, these were 58, 52 and $34 \%$, respectively. Median overall survival for ipilimumab monotherapy was recorded as 19.9 months (95\%CI: 16.9-24.9), nivolumab monotherapy as 37.6 months (95\%CI: 29.1 - not reached) and as not reached (95\% CI 38.2 months - not reached) for combination therapy arm. The objective response with combination therapy was noted in 58\% patients, $19 \%$ with ipilimumab monotherapy and $44 \%$ with nivolumab monotherapy whereas complete response was observed in 19,5 and $16 \%$ patients, respectively [56]. This data from CheckMate 067 allowed for confirmatory approval of combined therapy with nivolumab and ipilimumab for melanoma that initially received an accelerated approval by the FDA based upon the findings of CheckMate 069 trial. In addition to the above, the CheckMate 067 study also presented data on the influence of PD-L1 status on clinical outcomes. Patients with PD-L1 positive melanoma that received either combination therapy or nivolumab monotherapy had a median PFS of 14 . 0 months. However, study participants with PD-L1 negative melanoma exhibited a median PFS of 5.3 months (95\%CI: 2.8 to 7.1 ) with nivolumab monotherapy and 11 . 2 months (95\%CI: 8.0 - not reached) with combination therapy [55]. Lastly, with regards to the safety, this trial noted treatment related grade 3-4 adverse events in 59\% patients receiving nivolumab plus ipilimumab therapy, 
$21 \%$ of those receiving nivolumab only and $28 \%$ patients treated with ipilimumab monotherapy [56].

A different trial investigating the effects of variation in the sequence of administering different immunotherapy agents observed that the patient cohort receiving nivolumab before ipilimumab exhibited grade 3-5 treatment related adverse events (TRAE) in 50\% patients, response at 25 weeks in $41 \%$ and 12 months overall survival in 76\% patients (CheckMate 064, NCT01783938) [57]. In the patient cohort receiving ipilimumab prior to nivolumab, $42.8 \%$ patients had a grade $3-4$ TRAE, $20 \%$ patients exhibited response at 25 weeks and 12 months overall survival was noted in 54\% patients [57].

Finally, one trial investigated the safety and efficacy of combined therapy with reduced dose ipilimumab $(1 \mathrm{mg} /$ $\mathrm{kg}$ every 3 weeks for 4 doses) plus full-dose pembrolizu$\mathrm{mab}(2 \mathrm{mg} / \mathrm{kg}$ every 3 weeks) in patients with advanced melanoma (KEYNOTE-029, NCT02089685) [58]. This trial documented grade 1-2 and grade 3-4 treatment related adverse events in 51\% (78 of 153) and 45\% (69 of 153) patients, respectively. Immune-related adverse events were reported in 60\% (92 of 153) of melanoma patients, of which $27 \%$ (42 of 153) patients experienced one or more grade 3-4 immune-related adverse events. A number of study participants discontinued treatment due to treatment related adverse events. This included 14\% (22 of 153) patients discontinuing both ipilimumab and pembrolizumab, 9\% (14 of 153) patients stopping pembrolizumab only and 8\% (12 of 153) patients that discontinued ipilimumab only. With respect to survival outcomes (median 17.0 months follow-up), the study reported a PFS at 12 months of $69 \%$ (95\%CI: $60-75)$, objective response of 61\% (93 of 153 patients, 95\%CI: 53-69), complete response of $15 \%$ (23 of 153 patients), partial response of $46 \%$ (70 of 153 patients), stable disease in 18\% (28 of 153 patients) and progressive disease in 19\% (29 of 153 patients) [58]. These results were considerably better in terms of survival benefit and toxicity profile than combination therapy with nivolumab and ipilimumab for melanoma. Combined checkpoint inhibition with nivolumab $1 \mathrm{mg} / \mathrm{kg}$ plus ipilimumab $3 \mathrm{mg} / \mathrm{kg}$ evaluated in early phase 1 trials reported an objective response rate of 53\% (9 of 17 patients, 95\%CI: 28 to 77), complete response of $17 \%$ (3 of 17 patients), partial response of 35\% (6 of 17 patients) and serious treatment related adverse events in $49 \%$ patients (NCT01024231) [54, 59].

\section{Combination checkpoint inhibition trials in NSCLC}

Combined immunotherapy with anti-PD-1/PD-L1 and anti-CTLA-4 MoAbs in patients with NSCLC is currently being investigated in 16 ongoing trials (Table 2). Over half (10 of 16 trials) of these trials are evaluating combination therapy with nivolumab and ipilimumab with or without other therapeutic modalities. Other combination checkpoint inhibition regimens that are being examined in patients with NSCLC include tremelimumab plus durvalumab (5 of 16 trials) and pembrolizumab plus ipilimumab ( 1 of 16 trials).

Presently, five ongoing trials are assessing the combined use of anti-PD-1/PD-L1 and/or anti-CTLA-4 MoAbs with various chemotherapy agents. One of the trials has defined treatment cohorts on the basis of patient tumor marker status (NCT01998126). Patients with EGFR mutant NSCLC will receive erlotinib with either nivolumab or ipilimumab and those with $A L K$ rearranged NSCLC will be administered crizotinib with either nivolumab or ipilimumab. Another trial will compare PFS, ORR, and duration of response in patients with advanced NSCLC after administration of dasatinib (SRC-family protein-tyrosine kinase inhibitor) plus nivolumab, BMS-986016 (antiLAG-3 \{lymphocyte activation gene-3\} MoAb) plus nivolumab and ipilimumab plus nivolumab (NCT02750514) [10]. Participants enrolled in CheckMate 227 will be randomized to receive nivolumab plus platinum doublet chemotherapy (carboplatin/cisplatin plus gemcitabine for squamous NSCLC and carboplatin/cisplatin plus pemetrexed for non-squamous NSCLC) or combined therapy with nivolumab plus ipilimumab (NCT02477826). Similarly, CheckMate 722 will evaluate PFS for T790 M negative, EGFR mutant NSCLC patients treated with nivolumab plus platinum doublet chemotherapy (carboplatin/cisplatin plus pemetrexed) and ipilimumab plus nivolumab combination therapy (NCT02864251). The KEYNOTE 021 trial will focus on determining the RP2D for pembrolizumab plus ipilimumab, and it has a planned survival assessment to follow (NCT02039674). This trial will also evaluate the combined use of pembrolizumab with one or more standard chemotherapy agents using pre-defined treatment protocols. These include carboplatin, pemetrexed, paclitaxel, bevacizumab, erlotinib, and gefitinib (NCT02039674).

The development of a treatment regimen that integrates immunotherapy with PD-1/PD-L1 and CTLA-4 checkpoint inhibitors with surgery and radiation has been undertaken in one trial (NCT02696993). This trial will determine the RP2D for four combination therapies in NSCLC patients with brain metastases at the time of enrollment. The treatment regimens specified by the study protocol include nivolumab plus stereotactic radiosurgery, nivolumab plus ipilimumab and whole brain radiation therapy, nivolumab plus whole brain radiation therapy, and nivolumab plus ipilimumab and stereotactic radiosurgery.

At present, data on safety and survival benefit from combined immunotherapy with PD-1/PD-L1 and CTLA4 checkpoint inhibitors in NSCLC is available from three trials. One trial evaluated four experimental dosing schedules of combined therapy with nivolumab and ipilimumab against one monotherapy arm (nivolumab) in order to 
identify the regimen that delivers maximum clinical benefit with an acceptable adverse-effects profile (CheckMate 012, NCT01454102). In the patient cohort receiving ipilimumab $1 \mathrm{mg} / \mathrm{kg}$ every 6 weeks plus nivolumab $3 \mathrm{mg} / \mathrm{kg}$ every 2 weeks an ORR of 39\% was observed (95\%CI: $23-$ 55), median PFS was 3.9 months (95\%CI: 2.6-13.2), the 1 year OS rate was $69 \%$ (95\% CI: $52-81$ ), and grade $3-4$ treatment related adverse events (TRAE) occurred in 33\% of patients [60]. In another cohort, designated to receive ipilimumab $1 \mathrm{mg} / \mathrm{kg}$ every 12 weeks plus nivolumab $3 \mathrm{mg} / \mathrm{kg}$ every 2 weeks, reported grade 3-4 TRAE occurred in $37 \%$ of patients, the ORR was $47 \%$ (95\%CI: $31-$ 64), median PFS was 8.1 months (95\%CI: 5.6-13.6), and the 1 year OS rate was not calculated. Alternatively, patients treated with nivolumab $3 \mathrm{mg} / \mathrm{kg}$ every 2 weeks were noted to have an ORR of $23 \%$ (95\%CI: 13-37), median PFS of 3.6 months (95\%CI: 2.3-6.6), a 1 year OS rate of 73\% (95\% CI: 59-83), and TRAE occurred in 19\% of patients. The study also attempted to correlate the data for treatment response to tumor PD-L1 expression. In patients treated with nivolumab plus ipilimumab combination therapy, the ORR for tumors with PD-L1 expression $\geq 1 \%$ and $\geq 50 \%$ were 57 and $92 \%$, respectively. Conversely, patients receiving nivolumab monotherapy experienced an ORR of 28 and $50 \%$ for tumors with PD-L1 expression of $\geq 1 \%$ and $\geq 50 \%$, respectively. Based on the overall analysis, treatment with ipilimumab $1 \mathrm{mg} / \mathrm{kg}$ every 6 weeks plus nivolumab $3 \mathrm{mg} / \mathrm{kg}$ every 2 weeks was selected for further investigation [60]. This regimen is presently under evaluation in the CheckMate 227 trial (NCT02477826).

The KEYNOTE 021 trial evaluated the combined use of pembrolizumab and traditional chemotherapy; it noted an ORR of 52\% (13 of 25 patients, $95 \% \mathrm{CI}$ : 31 to 72 ) and PFS of 10 months (95\%CI: 4 - not reached) in patients treated with carboplatin $6 \mathrm{mg} /$ $\mathrm{mL} / \mathrm{min}$ plus pembrolizumab 2 or $10 \mathrm{mg} / \mathrm{kg}$ and paclitaxel $200 \mathrm{mg} / \mathrm{m}^{2}$ (NCT02039674) [61]. Likewise, the ORR was $48 \%$ (12 of 25 patients, $95 \%$ CI: 28 to 69 ) and PFS was "not reached" (95\%CI: 4 - not reached) in patients that received paclitaxel $200 \mathrm{mg} / \mathrm{m}^{2}$ plus pembrolizumab 2 or $10 \mathrm{mg} / \mathrm{kg}$, carboplatin $6 \mathrm{mg} / \mathrm{mL} /$ min and bevacizumab $15 \mathrm{mg} / \mathrm{kg}$. Another cohort of patients that were given pemetrexed $500 \mathrm{mg} / \mathrm{m}^{2}$ plus carboplatin $5 \mathrm{mg} / \mathrm{mL} / \mathrm{min}$ and pembrolizumab 2 or $10 \mathrm{mg} / \mathrm{kg}$ were noted to have an ORR of $71 \%$ (17 of 24 patients, $95 \% \mathrm{CI}$ : 49 to 87 ) and PFS of 10 months (95\%CI: 6 to 15$)$ [61].

A different trial assessing combination checkpoint inhibition in patients with NSCLC involved the use of various doses of tremelimumab $(1,3$ or $10 \mathrm{mg} / \mathrm{kg})$ in combination with durvalumab $(3,10,15$ or $20 \mathrm{mg} / \mathrm{kg}$ ) during the dose escalation phase (NCT02000947). The objective response (investigator assessed) in the tremelimumab $1 \mathrm{mg} / \mathrm{kg}$ treatment cohort was 23\% (6 of 26 patients, 95\%CI: 9 to 44) [62]. Also, an objective response was noted in 22\% (2 of 9 patients, 95\%CI: 3 to 60 ) of cases with PD-L1 positive tumors and 29\% (4 of 14 patients, $95 \% \mathrm{CI}$ : 8 to 58 ) of cases with PD-L1 negative tumors. MTD was exceeded for treatment with tremelimumab $3 \mathrm{mg} / \mathrm{kg}$ plus durvalumab $20 \mathrm{mg} / \mathrm{kg}$, with almost 30\% (2 of 6 patients) of patients reporting DLTs [62].

\section{Discussion}

There is great enthusiasm surrounding combination immunotherapy with PD-1/PD-L1 and CTLA-4 checkpoint inhibitors. The superior outcomes with combined immunotherapy over single-agent regimens in preclinical studies, together with the approval of nivolumab plus ipilimumab combination therapy for patients with melanoma have shed light on the therapeutic potential of this concept. The possibility of expanding the spectrum of indications for combination checkpoint inhibition to a wide range of tumor histologies is being explored in several trials. Simultaneously, extensive efforts have been undertaken to optimize clinical benefit to adverse effects ratios with combination checkpoint inhibition.

Combined therapy with anti-PD-1/PD-L1 and antiCTLA-4 MoAbs in advanced melanoma has exhibited better survival outcomes in comparison with singleagent immunotherapy (Fig. 3). CheckMate 067 reported a survival benefit with nivolumab plus ipilimumab combination therapy (median PFS: 11.5 months, objective response $58 \%$ ) over monotherapy with ipilimumab (median PFS: 2.9 months, objective response 19\%) and nivolumab (median PFS: 6.9 months, objective response $44 \%)$ [55, 56, 63]. It is, however, important to note that though this study met the coprimary end-point of exhibiting improved overall survival with combination therapy versus ipilimumab, it was underpowered to reflect upon the use of nivolumab and ipilimumab combination therapy over antiPD-1 monotherapy followed by subsequent ipilimumab rescue. Another trial, CheckMate 069, recorded the hazard ratio for death or progression of disease for combined versus single-agent immunotherapy for $B R A F$ wild-type as 0.40 (95\% CI: 0.23 to $0.68, p<0$. 001 ) and BRAF-mutant as 0.38 (95\% CI: 0.15 to 1.00 ) melanoma patients, underscoring the survival benefit with combination checkpoint inhibition [53]. Akin to the aforementioned trials, several ongoing trials will compare single-agent immunotherapy with combination checkpoint inhibition with anti-PD-1/PD-L1 plus anti-CTLA-4 checkpoint inhibitors (NCT0194080 9, NCT02736123, NCT02519322, NCT02731729, NC T02374242, NCT02523313, NCT02599402, NCT02460 068, NCT02750514, NCT02154490, NCT02785952, 


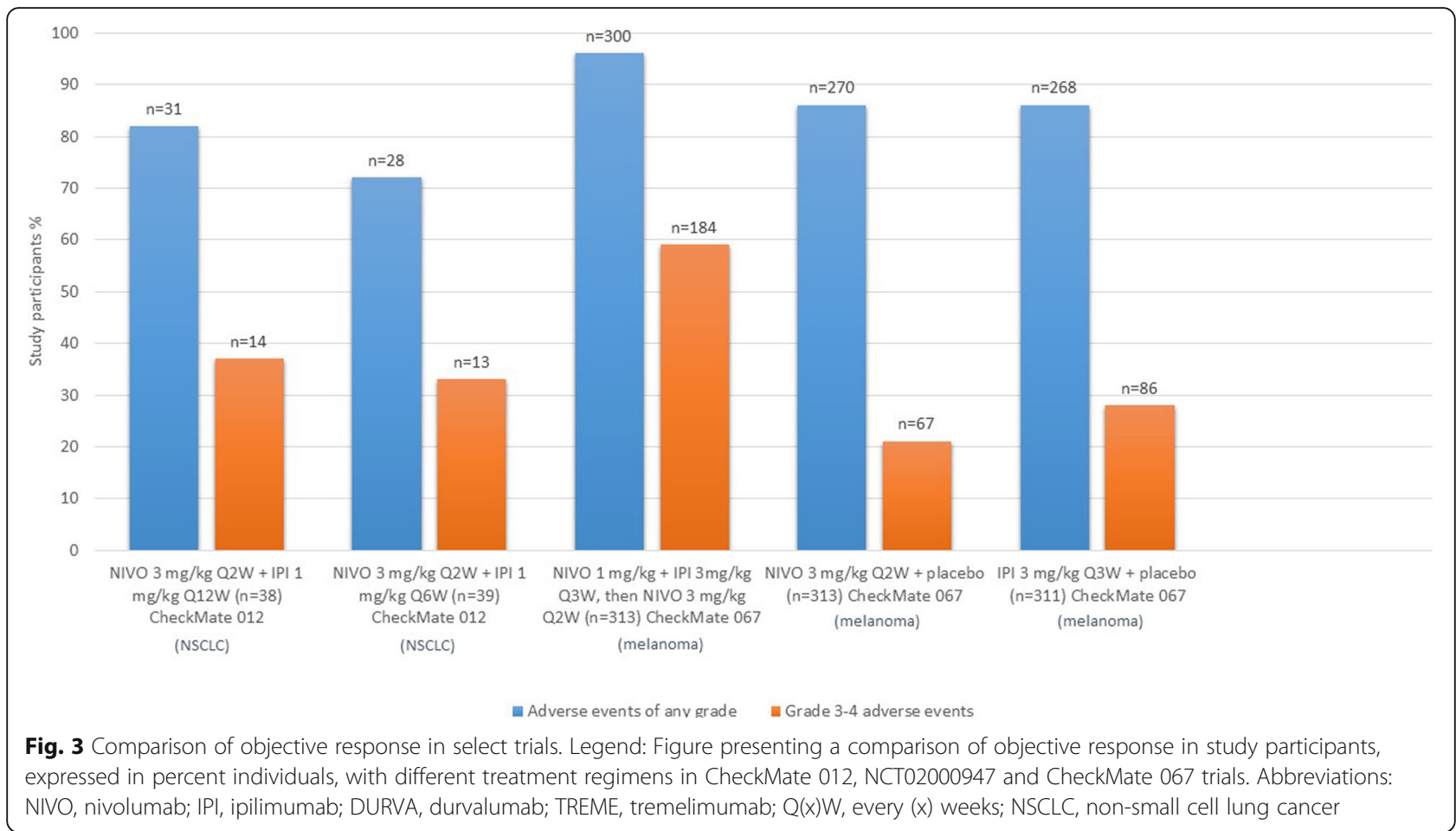

NCT02477826, NCT02352948, NCT02453282, NCT01 928394, NCT02537418).

Several studies have attempted to correlate the expression of PD-L1 and response to combination checkpoint inhibition with anti-PD-1/PD-L1 plus anti-CTLA-4 MoAbs. PD-L1 positivity has classically been defined as visualization of at least $5 \%$ of tumor cells with PD-L1 staining in a section containing a minimum of one hundred cells suitable for evaluation [53, 54, 64, 65]. NCT01024231 documented response with combination therapy regardless of tumor PD-L1 status [54]. Likewise, CheckMate 069 reported no significant variation in response to combination checkpoint inhibition based on PD-L1 status [53]. Interestingly, patients with PD-L1 positive melanoma had better objective response rates as compared to those who tested negative for PD-L1 with ipilimumab monotherapy [53]. Another trial, CheckMate 067, noted a considerable advantage in median PFS with combined ipilimumab plus nivolumab (11.2 months) as opposed to nivolumab monotherapy (5.3 months) in patients with PD-L1 negative melanoma. The median PFS for patients with PD-L1 positive tumors, however, was the same for both treatment groups (14 months) [65]. Similarly, a different trial evaluating low-dose ipilimumab plus nivolumab in NSCLC patients reported an ORR of $57 \%$ in patients with tumor PD-L1 expression $\geq 1$ and $92 \%$ in patients with tumor PD-L1 expression $\geq 50 \%$ (CheckMate 012) [60]. Considering the above, it appears that a pragmatic approach for selecting the most appropriate immunotherapy regimen (monotherapy versus combination therapy) may be through a novel treatment algorithm including tumor PD-L1 status.

The incidence of treatment-related adverse events with combination checkpoint inhibition has been a matter of concern in pivotal trials. The CheckMate 069 trial, which evaluated combination therapy with nivolumab and ipilimumab (nivolumab $1 \mathrm{mg} / \mathrm{kg}$ plus ipilimumab $3 \mathrm{mg} / \mathrm{kg}$ every 3 weeks for 4 doses followed by monotherapy with nivolumab $3 \mathrm{mg} / \mathrm{kg}$ every 2 weeks) versus ipilimumab monotherapy in melanoma patients, documented treatment-associated grade 3-4 adverse events in $54 \%$ of patients receiving combination therapy as compared to $24 \%$ receiving ipilimumab monotherapy [53]. These results suggest that melanoma patients receiving combination checkpoint inhibition were much more likely to develop severe drug-related adverse events as compared to treatment with ipilimumab monotherapy. Although $68 \%$ of patients that discontinued combination therapy due to toxicity exhibited an objective response, concerns over treatment-related toxicity with combination checkpoint inhibition regimens persuade some to favor immunecheckpoint inhibitor monotherapy [53]. In order to validate these findings, the same combination therapy regimen was evaluated in the CheckMate 067 trial and compared ipilimumab monotherapy and nivolumab monotherapy in parallel arms [56]. The frequency of grade 3-4 adverse events in patients treated with combination therapy (59\% patients) was higher than that recorded for patients receiving monotherapy with ipilimumab (28\% patients) or nivolumab (21\% patients) [56]. However, treatment related adverse events 
with combination therapy were described as manageable and the study concluded that this regimen was suitable for further investigation. Considering the above, it may be stated that one should be cautious in selection of combination immune checkpoint inhibition over monotherapy in elderly patients with high frailty index.

A retrospective pooled analysis conducted to study efficacy and safety of combined therapy with nivolumab and ipilimumab in patients that discontinued therapy due to adverse events presented interesting findings. Data from phase 2 and phase 3 trials for advanced melanoma evaluating nivolumab $1 \mathrm{mg} / \mathrm{kg}$ plus ipilimumab $3 \mathrm{mg} / \mathrm{kg}$ every 3 weeks followed by monotherapy with nivolumab $3 \mathrm{mg} / \mathrm{kg}$ every 2 weeks, was pooled to compare outcomes in participants who discontinued therapy due to adverse events versus those who did not [66]. After a minimum of 18 months follow-up, median PFS and objective response rate for patients that discontinued therapy during induction were found to be 8 . 4 months and $58.3 \%$ respectively. On the other hand, patients that did not discontinue therapy were found to have a median PFS and objective response rate of 10 . 8 months and $50.2 \%$, respectively. The nearly similar efficacy outcomes in the two groups indicated that despite discontinuing the therapy, patients continued to derive benefits from the treatment [66]. In other words, shorter course of treatment exhibited outcomes comparable to those achieved with full-course combination therapy. Also, it may be argued that immune related adverse events could possibly serve as surrogate markers for patients that may benefit from immunotherapy regimens.

The use of low-dose combination checkpoint inhibition appears to be a promising approach for improving clinical benefit without significantly increasing adverse events. The CheckMate 012 trial assessed several nivolumab plus ipilimumab combination regimens as first-line therapy in NSCLC patients. The frequency of treatmentassociated grade 3-4 adverse events with nivolumab $3 \mathrm{mg} / \mathrm{kg}$ every 2 weeks plus ipilimumab $1 \mathrm{mg} / \mathrm{kg}$ every 12 weeks was $37 \%$ and with nivolumab $3 \mathrm{mg} / \mathrm{kg}$ every 2 weeks plus ipilimumab $1 \mathrm{mg} / \mathrm{kg}$ every 6 weeks was $33 \%$ [60]. The frequency of treatment-related grade 3-4 adverse events with nivolumab monotherapy was $19 \%$ [60]. Low-dose combination checkpoint inhibition with nivolumab and ipilimumab had a more acceptable adverse-effects profile compared to the combination regimen evaluated in CheckMate 067 (Fig. 4). However, it may be argued that the variation in toxicity profile was secondary to, or at least in part influenced by a difference in tumor histologies. One example may be the FDA

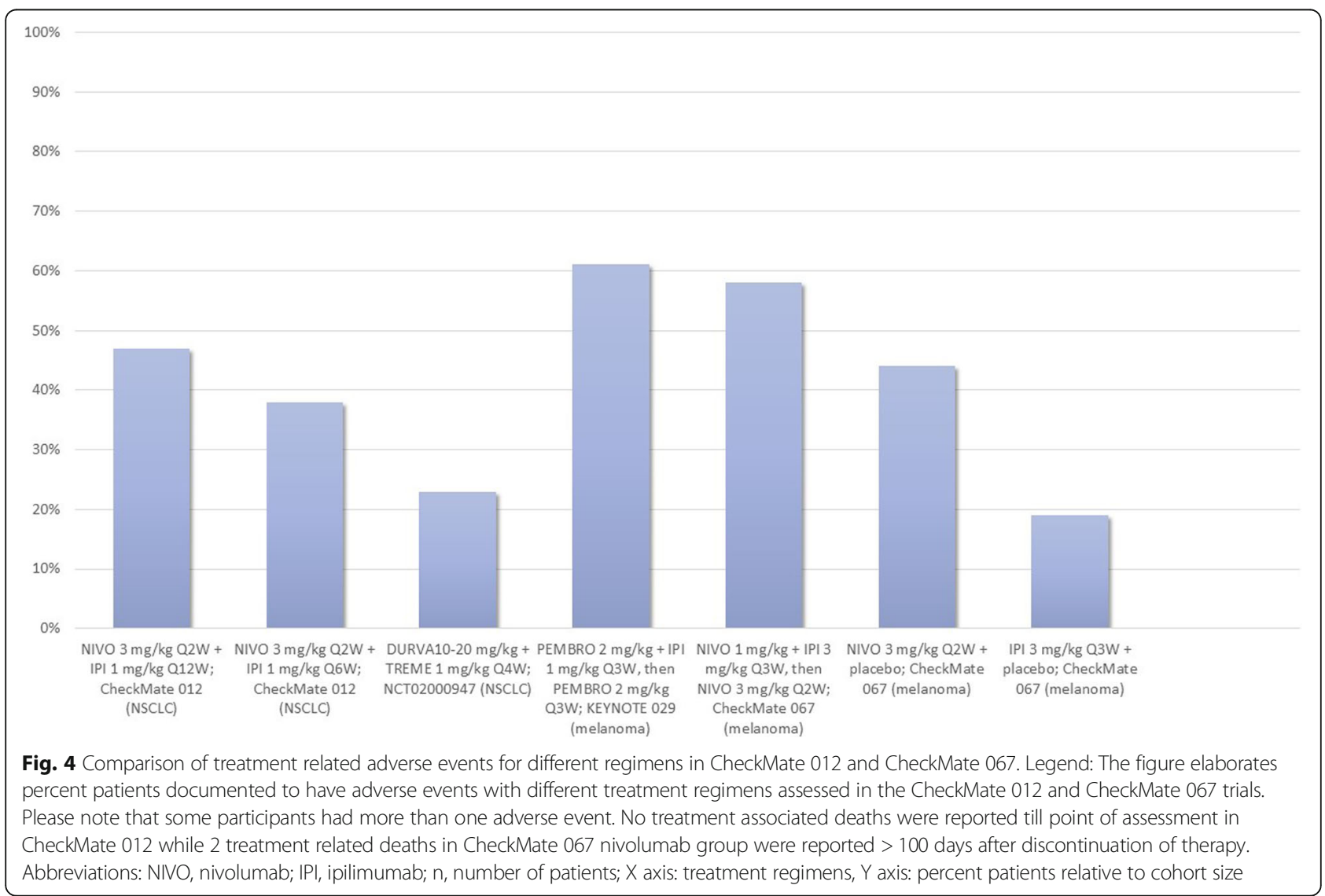


approved regimen for melanoma (4 cycles of ipilimumab $3 \mathrm{mg} / \mathrm{kg}$ plus nivolumab $1 \mathrm{mg} / \mathrm{kg}$ every 3 weeks followed by nivolumab $240 \mathrm{mg}$ every 2 weeks), that exhibited an acceptable safety profile and response in recurrent small cell lung cancer but at the same time demonstrated a poor safety and efficacy profile in NSCLC, leading to abandonment of this strategy in the latter [60, 67]. Data from ongoing studies investigating low-dose combination checkpoint inhibition in a number of tumor histologies will be crucial to the validation of these findings.

Another step towards achieving the right balance of efficacy and incidence of adverse events may be to critically assess the use of common terminology criteria for adverse events (CTCAE). CTCAE are used for documenting chemotherapy associated adverse events [68]. They are instrumental to determine the appropriate dose limiting toxicity for the experimental regimen in a trial. This in-turn has a significant bearing on the recommended phase 2 dose for the novel therapeutic agent. CTCAE are, however, now being applied to immune related adverse events for novel immunotherapy regimens [69]. A phase 1 trial investigating nivolumab plus ipilimumab in melanoma patients used an asymptomatic rise in lipase as the primary dose limiting toxicity, central to informing the recommended phase 2 dose in this trial. A retrospective study analyzed the association between asymptomatic rise in lipase and amylase (grade 3 and above) with pancreatitis in 119 participants and found only 2 patients to have pancreatitis. This represented $6.3 \%$ of all patients with grade 3 and above lipase and $20 \%$ of those with grade 3 or above increase in both amylase and lipase. Thus, in simple terms, lipase did not appear to be a relevant marker for pancreatitis. This observation thus signifies the need to exercise appropriate caution when grading independent lab values using CTCAE in immunotherapy trials [69].

Existing evidence suggests that single-agent immunotherapy for tumors with high PD-L1 expression ( $\geq 50 \%$ cells positive for PD-L1 staining) can achieve far superior outcomes than chemotherapy in similar settings. In patients with NSCLC with high PD-L1 expression, pembrolizumab exhibited a response rate of 45\%, PFS of 10 . 3 months, and a 1-year survival rate of 70\% [70]. In comparison, treatment with standard of care chemotherapy had a response rate of $28 \%$, PFS of 6 months, and 1year survival rate of 54\% (KEYNOTE 026) [70]. On the other hand, trials involving a lower cut-off value for PDL1 positivity ( $\geq 5 \%$ cells positive for PD-L1 staining) failed to demonstrate any advantage in clinical efficacy with single-agent immunotherapy over standard chemotherapy (CheckMate 026) [71, 72]. Reflecting on the data on efficacy and overall toxicity profile of single-agent immunotherapy regimens in tumors with high PD-L1 expression, outperforming these regimens may prove to be a challenge for combination checkpoint inhibition regimens. Further data from ongoing trials will be vital to conclusively determine if single-agent immunotherapy with pembrolizumab can be replaced with a combined immunotherapy regimen.

Immunotherapy with PD-1/PD-L1 plus CTLA-4 checkpoint inhibitors for a diverse set of solid tumors is currently being investigated in 8 trials (Table 3 ). Half of all phase 1/phase 2 solid tumor trials are evaluating combined therapy with nivolumab and ipilimumab. Others include three trials with combined therapy with tremelimumab plus durvalumab and one trial with atezolizumab plus ipilimumab. Each of these eight trials will evaluate combination checkpoint inhibition regimens in a large number of malignancies. The data gathered from these studies will be crucial to identifying tumor histologies that would benefit most from combination checkpoint inhibition.

Recently, the FDA approved the use of pembrolizumab for unresectable/metastatic mismatch repair deficient (dMMR) or microsatellite instability-high (MSI-H) solid tumors and colorectal cancer with progression on prior therapy. This was based upon data from KEYNOTE 012, KEYNOTE 028, KEYNOTE 164, KEYNOTE 016 and KEYNOTE 158 [73-76]. The advised regimen is pembrolizumab $10 \mathrm{mg} / \mathrm{kg}$ every 2 weeks or $200 \mathrm{mg}$ every 3 weeks for up to 24 months, unacceptable toxicity or progression of disease. It is of note that this is the first time when a drug has been approved not on the basis of tumor location but a tumor biomarker.

The Dual Anti-CTLA-4 and Anti-PD-1 Blockade in Rare Tumors (DART) trial will evaluate response to combination checkpoint inhibition for a large number of rare tumors in a basket fashion (NCT02834013). In this trial, minimizing toxicity profiles without compromising clinical efficacy is the primary goal. We nominated the low-dose combination therapy with fixed-dose nivolumab and wider interval ipilimumab (nivolumab $240 \mathrm{mg}$ every 2 weeks plus ipilimumab $1 \mathrm{mg} / \mathrm{kg}$ every 6 weeks) for assessment in this trial. Our treatment regimen is based on the superior toxicity profile for this regimen observed in CheckMate 012 as compared to the FDA approved combination therapy regimen (nivolumab $1 \mathrm{mg} / \mathrm{kg}$ plus ipilimumab $3 \mathrm{mg} / \mathrm{kg}$ every 3 weeks for 4 doses followed by monotherapy with nivolumab $3 \mathrm{mg} / \mathrm{kg}$ every 2 weeks) in CheckMate 069 [53, 60]. Based on the fact that no significant difference in response was recorded between PD-L1 positive and negative patients receiving combination therapy, we decided to recruit study participants irrespective of tumor PD-L1 status. Through this trial, we expect to provide critical data for expanding the application of low-dose combination therapy in rare tumors. 


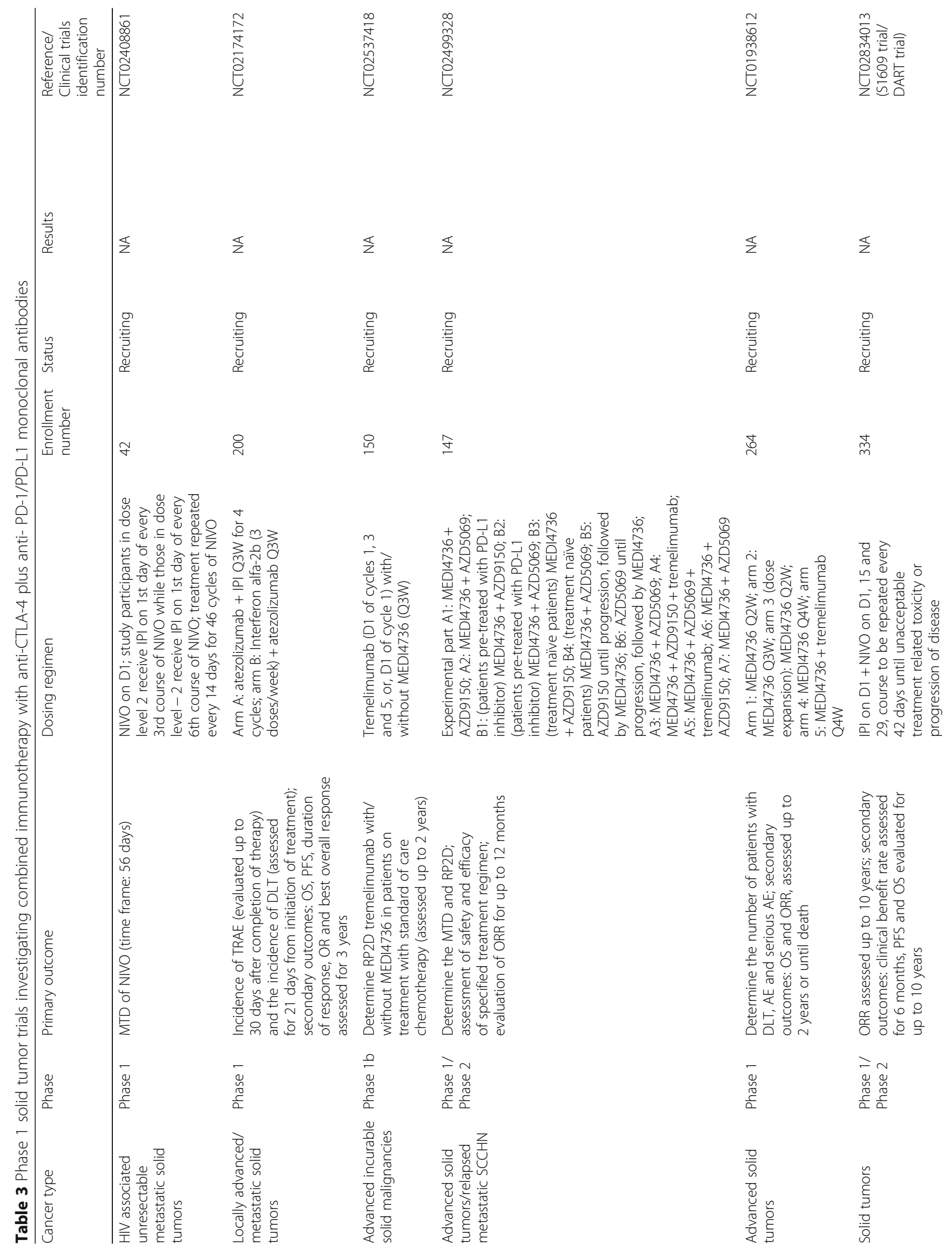




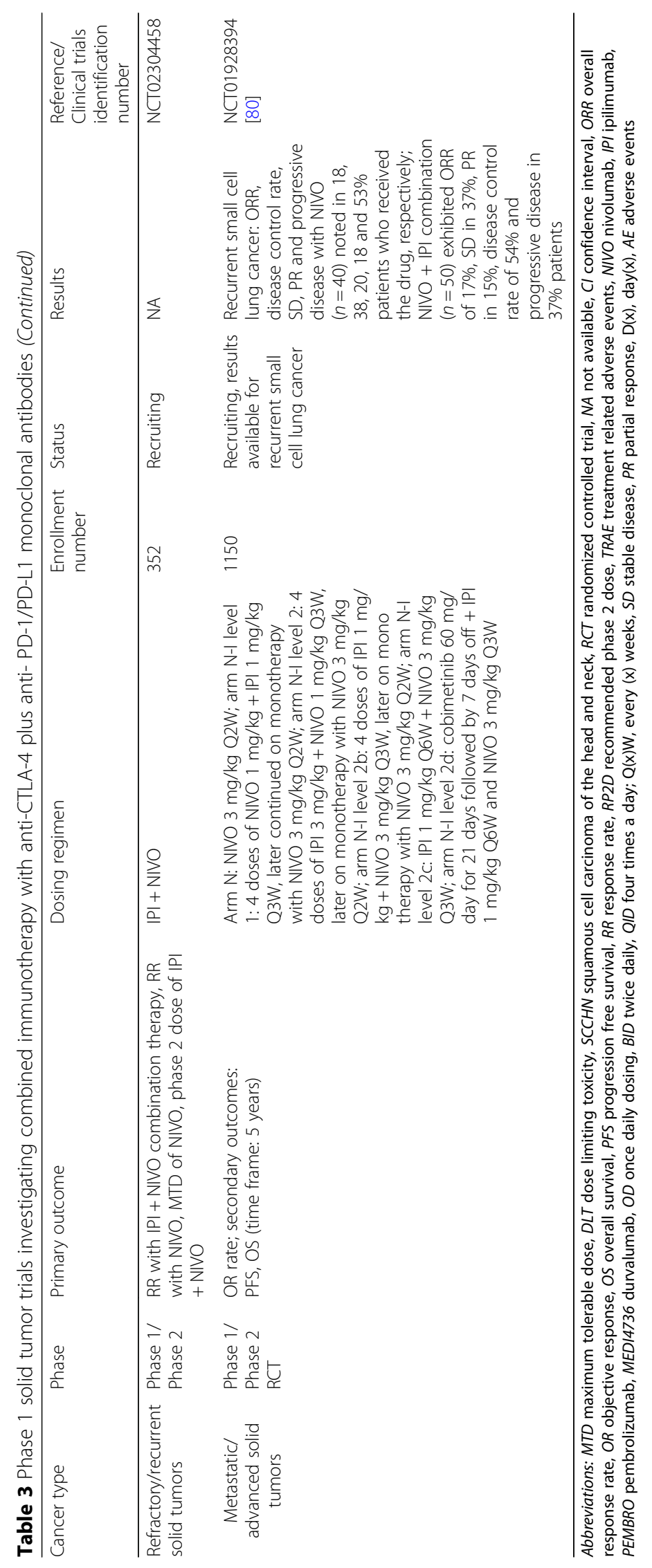


Recent studies have suggested that sequential administration of immune-checkpoint inhibitors targeting various pathways may benefit cancer patients exhibiting treatment resistance. A multi-center retrospective study evaluated outcomes with ipilimumab and combination therapy with nivolumab and ipilimumab in advanced melanoma patients that previously failed treatment with anti-PD-1 MoAbs [77]. Patients receiving ipilimumab monotherapy were observed to have superior disease control as compared to those receiving combination checkpoint inhibition (42\% versus 33\%) [77]. Of note, though this was a retrospective study, it was insufficiently powered to detect the difference. A different retrospective analysis of 10 melanoma patients that received ipilimumab after progression on anti-PD-1 therapy found that 1 of 10 patients exhibited partial remission and an additional four patients had stable disease [78]. Similar to the findings of the above and other retrospective studies, the CheckMate 064 documented a higher treatment efficacy in melanoma patients receiving nivolumab prior to ipilimumab versus those receiving ipilimumab prior to nivolumab [57]. However, superior clinical efficacy with the former regimen was associated with an inferior toxicity profile to the latter [57]. Of note, the trial involved a planned switch to reverse sequence at 12 weeks and not at progression. Therefore, it remains unclear if switching to combinatorial regimen at the time of progression is a feasible approach. The outcomes from NCT02731729 trial should perhaps be able to provide some direction on this matter.

\section{Conclusion}

Combination immunotherapy is evolving at a phenomenal pace. In light of initial success in patients with melanoma, efforts to explore the indications for combination checkpoint inhibition with anti-PD-1/PDL1 and anti-CTLA-4 MoAbs have diversified to a large number of tumor histologies. Several treatment strategies intended for achieving better clinical efficacy and to overcome challenges such as treatment resistance and toxicity associated with the use of immunotherapy agents, are presently under investigation. Of note, the use of low-dose combination checkpoint inhibition with nivolumab and ipilimumab in NSCLC appears to be a promising approach. Alternatively, the use of nivolumab prior to ipilimumab in the induction phase for melanoma patients may be a simple but effective strategy to achieve superior outcomes. Data from ongoing trials is expected to provide vital evidence for validation of the above preliminary findings and facilitate the application of combination checkpoint inhibition on a larger scale.

Availability of data and materials

All data analyzed during this study is included in this published article.
Authors' contributions

YKC and AA gathered, analyzed and interpreted the data from various published articles. All authors read and approved the final manuscript.

Ethics approval and consent to participate Not applicable.

\section{Competing interests}

The authors declare that they have no competing interests.

\section{Publisher's Note}

Springer Nature remains neutral with regard to jurisdictional claims in published maps and institutional affiliations.

\section{Author details}

${ }^{1}$ Developmental Therapeutics Program of the Division of Hematology Oncology, Early Phase Clinical Trials Unit, 645 N. Michigan Avenue, Suite 1006, Chicago, IL 60611, USA. ${ }^{2}$ Robert H. Lurie Comprehensive Cancer Center of Northwestern University, 645 N. Michigan Avenue, Suite 1006, Chicago, IL 60611 , USA. ${ }^{3}$ Northwestern University Feinberg School of Medicine, $645 \mathrm{~N}$. Michigan Avenue, Suite 1006, Chicago, IL 60611, USA.

Received: 1 October 2017 Accepted: 2 May 2018

Published online: 16 May 2018

\section{References}

1. Boyiadzis M, Foon KA. Approved monoclonal antibodies for cancer therapy. Expert Opin Biol Ther. 2008;8(8):1151-8.

2. Scott AM, Wolchok JD, Old LJ. Antibody therapy of cancer. Nat Rev Cancer. 2012;12(4):278-87.

3. Chames P, Van Regenmortel M, Weiss E, Baty D. Therapeutic antibodies: successes, limitations and hopes for the future. Br J Pharmacol. 2009;157(2):220-33.

4. Reslan L, Dalle S, Dumontet C. Understanding and circumventing resistance to anticancer monoclonal antibodies. Taylor \& Francis. MAbs. 2009;1 (3):222-29. https://doi.org/10.4161/mabs.1.3.8292.

5. Pillay V, Gan HK, Scott AM. Antibodies in oncology. New Biotechnol. 2011; 28(5):518-29.

6. McKay RR, Choueiri TK: Emerging role of combination immunotherapy regimens in metastatic renal cell carcinoma. In: ASCO Annual Meeting: 2016.

7. Curran MA, Montalvo W, Yagita H, Allison JP. PD-1 and CTLA-4 combination blockade expands infiltrating $T$ cells and reduces regulatory $T$ and myeloid cells within B16 melanoma tumors. Proc Natl Acad Sci. 2010;107(9):4275-80.

8. Chen L, Han X. Anti-PD-1/PD-L1 therapy of human cancer: past, present, and future. J Clin Invest. 2015;125(9):3384-91.

9. DiGiulio S. FDA approves Opdivo-Yervoy combo for melanoma, first combo immunotherapy regimen for Cancer. Oncology Times. 2015. https://journals.lww. com/oncology-times/blog/fdaactionsandupdates/pages/post.aspx?PostlD=116.

10. Dictionary ND. National Cancer Institute at the National Institutes of Health. https://www.cancer.gov/publications/dictionaries.

11. Buchbinder El, Desai A. CTLA-4 and PD-1 pathways: similarities, differences, and implications of their inhibition. Am J Clin Oncol. 2016;39(1):98-106.

12. Collins AV, Brodie DW, Gilbert RJ, laboni A, Manso-Sancho R, Walse B, Stuart DI, van der Merwe PA, Davis SJ. The interaction properties of costimulatory molecules revisited. Immunity. 2002;17(2):201-10.

13. Chambers CA, Kuhns MS, Egen JG, Allison JP. CTLA-4-mediated inhibition in regulation of $T$ cell responses: mechanisms and manipulation in tumor immunotherapy. Annu Rev Immunol. 2001;19(1):565-94.

14. Keir ME, Butte MJ, Freeman GJ, Sharpe AH. PD-1 and its ligands in tolerance and immunity. Annu Rev Immunol. 2008;26:677-704.

15. Egen JG, Kuhns MS, Allison JP. CTLA-4: new insights into its biological function and use in tumor immunotherapy. Nat Immunol. 2002;3(7):611-8.

16. Parry RV, Chemnitz JM, Frauwirth KA, Lanfranco AR, Braunstein I, Kobayashi SV, Linsley PS, Thompson CB, Riley JL. CTLA-4 and PD-1 receptors inhibit Tcell activation by distinct mechanisms. Mol Cell Biol. 2005;25(21):9543-53.

17. Masteller EL, Chuang E, Mullen AC, Reiner SL, Thompson CB. Structural analysis of CTLA-4 function in vivo. J Immunol. 2000;164(10):5319-27.

18. Krummel MF, Allison JP. CD28 and CTLA-4 have opposing effects on the response of T cells to stimulation. J Exp Med. 1995;182(2):459-65.

19. Linsley PS, Bradshaw J, Greene J, Peach R, Bennett KL, Mittler RS. Intracellular trafficking of CTLA-4 and focal localization towards sites of TCR engagement. Immunity. 1996;4(6):535-43. 
20. Takahashi T, Tagami T, Yamazaki S, Uede T, Shimizu J, Sakaguchi N, Mak TW, Sakaguchi S. Immunologic self-tolerance maintained by CD25+ CD4+ regulatory $T$ cells constitutively expressing cytotoxic $T$ lymphocyteassociated antigen 4. J Exp Med. 2000;192(2):303-10.

21. Ishida Y, Agata Y, Shibahara K, Honjo T. Induced expression of PD-1, a novel member of the immunoglobulin gene superfamily, upon programmed cell death. EMBO J. 1992;11(11):3887.

22. Chen L. Co-inhibitory molecules of the B7-CD28 family in the control of Tcell immunity. Nat Rev Immunol. 2004;4(5):336-47.

23. Taube JM, Klein A, Brahmer JR, Xu H, Pan X, Kim JH, Chen L, Pardoll DM, Topalian SL, Anders RA. Association of PD-1, PD-1 ligands, and other features of the tumor immune microenvironment with response to antiPD-1 therapy. Clin Cancer Res. 2014;20(19):5064-74.

24. Keir ME, Liang SC, Guleria I, Latchman YE, Qipo A, Albacker LA, Koulmanda M, Freeman GJ, Sayegh MH, Sharpe AH. Tissue expression of PD-L1 mediates peripheral T cell tolerance. J Exp Med. 2006;203(4):883-95.

25. Tseng S-Y, Otsuji M, Gorski K, Huang X, Slansky JE, Pai SI, Shalabi A, Shin T, Pardoll DM, Tsuchiya H. B7-DC, a new dendritic cell molecule with potent costimulatory properties for T cells. J Exp Med. 2001;193(7):839-46.

26. Zitvogel L, Kroemer G. Targeting PD-1/PD-L1 interactions for cancer immunotherapy. Oncoimmunol. 2012;1(8):1223-5.

27. Dong H, Strome SE, Salomao DR, Tamura H, Hirano F, Flies DB, Roche PC, Lu J, Zhu G, Tamada K. Tumor-associated B7-H1 promotes T-cell apoptosis: a potential mechanism of immune evasion. Nat Med. 2002;8(8):793-800.

28. Iwai Y, Ishida M, Tanaka Y, Okazaki T, Honjo T, Minato N. Involvement of PD-L1 on tumor cells in the escape from host immune system and tumor immunotherapy by PD-L1 blockade. Proc Natl Acad Sci. 2002;99(19):12293-7.

29. Tsushima F, Yao S, Shin T, Flies A, Flies S, Xu H, Tamada K, Pardoll DM, Chen $\mathrm{L}$. Interaction between $\mathrm{B} 7-\mathrm{H} 1$ and PD-1 determines initiation and reversal of T-cell anergy. Blood. 2007;110(1):180-5.

30. Duraiswamy J, Kaluza KM, Freeman GJ, Coukos G. Dual blockade of PD-1 and CTLA-4 combined with tumor vaccine effectively restores T-cell rejection function in tumors. Cancer Res. 2013;73(12):3591-603.

31. Reiman JM, Kmieciak M, Manjili MH, Knutson KL. Tumor immunoediting and immunosculpting pathways to cancer progression. In: Seminars in Cancer Biology. Elsevier; 2007;17(4):275-87. https://www.sciencedirect.com/science/ article/pii/S1044579X0700034X.

32. Dunn GP, Bruce AT, Ikeda H, Old L, Schreiber RD. Cancer immunoediting: from immunosurveillance to tumor escape. Nat Immunol. 2002;3(11):991-8.

33. Houghton AN, Guevara-Patiño JA. Immune recognition of self in immunity against cancer. J Clin Invest. 2004;114(4):468-71.

34. Schreiber RD, Old LJ, Smyth MJ. Cancer immunoediting: integrating immunity's roles in cancer suppression and promotion. Science. 2011; 331(6024):1565-70.

35. Kim R, Emi M, Tanabe K. Cancer immunoediting from immune surveillance to immune escape. Immunology. 2007;121(1):1-14.

36. Beatty $\mathrm{GL}$, Gladney WL. Immune escape mechanisms as a guide for cancer immunotherapy. Clin Cancer Res. 2015;21(4):687-92.

37. Drake C. Combination immunotherapy approaches. Ann Oncol. 2012; 23(suppl 8):viii41-6.

38. Poschke I, Mougiakakos D, Kiessling R. Camouflage and sabotage: tumor escape from the immune system. Cancer Immunol Immunother. 2011;60(8):1161-71.

39. Swann JB, Smyth MJ. Immune surveillance of tumors. J Clin Invest. 2007; 117(5):1137-46.

40. Galluzzi L, Vacchelli E, Pedro B-S, Buqué A, Senovilla L, Baracco EE, Bloy N, Castoldi F, Abastado J-P, Agostinis P. Classification of current anticancer immunotherapies. Oncotarget. 2015;5:12472-508.

41. Ferris RL, Blumenschein G Jr, Fayette J, Guigay J, Colevas AD, Licitra L, Harrington K, Kasper S, Vokes EE, Even C. Nivolumab for recurrent squamous-cell carcinoma of the head and neck. N Engl J Med. 2016; 2016(375):1856-67.

42. Motzer RJ, Rini BI, McDermott DF, Redman BG, Kuzel TM, Harrison MR, Vaishampayan UN, Drabkin HA, George S, Logan TF. Nivolumab for metastatic renal cell carcinoma: results of a randomized phase II trial. J Clin Oncol. 2015;33(13):1430-7.

43. Borghaei H, Paz-Ares L, Horn L, Spigel DR, Steins M, Ready NE, Chow LQ, Vokes EE, Felip E, Holgado E. Nivolumab versus docetaxel in advanced nonsquamous non-small-cell lung cancer. N Engl J Med. 2015;373(17):1627-39.

44. Brahmer J, Reckamp KL, Baas P, Crinò L, Eberhardt WE, Poddubskaya E, Antonia S, Pluzanski A, Vokes EE, Holgado E. Nivolumab versus docetaxel in advanced squamous-cell non-small-cell lung cancer. N Engl J Med. 2015;373(2):123-35.
45. Weber JS, D'Angelo SP, Minor D, Hodi FS, Gutzmer R, Neyns B, Hoeller C, Khushalani NI, Miller WH, Lao CD. Nivolumab versus chemotherapy in patients with advanced melanoma who progressed after anti-CTLA-4 treatment (CheckMate 037): a randomised, controlled, open-label, phase 3 trial. Lancet Oncol. 2015;16(4):375-84.

46. Ansell SM, Lesokhin AM, Borrello I, Halwani A, Scott EC, Gutierrez M, Schuster SJ, Millenson MM, Cattry D, Freeman GJ. PD-1 blockade with nivolumab in relapsed or refractory Hodgkin's lymphoma. N Engl J Med. 2015;372(4):311-9.

47. Garon EB, Rizvi NA, Hui R, Leighl N, Balmanoukian AS, Eder JP, Patnaik A, Aggarwal C, Gubens M, Horn L, et al. Pembrolizumab for the treatment of non-small-cell lung Cancer. N Engl J Med. 2015;372(21):2018-28.

48. Robert C, Schachter J, Long GV, Arance A, Grob JJ, Mortier L, Daud A, Carlino MS, McNeil C, Lotem M, et al. Pembrolizumab versus Ipilimumab in Advanced Melanoma. N Engl J Med. 2015;372(26):2521-32.

49. Seiwert TY, Haddad Rl, Gupta S, Mehra R, Tahara M, Berger R, Lee S-H, Burtness B, Le DT, Heath K. Antitumor activity and safety of pembrolizumab in patients (pts) with advanced squamous cell carcinoma of the head and neck (SCCHN): preliminary results from KEYNOTE-012 expansion cohort. In: ASCO annual meeting proceedings; 2015. p. LBA6008.

50. Rosenberg JE, Hoffman-Censits J, Powles T, van der Heijden MS, Balar AV, Necchi A, Dawson N, O'Donnell PH, Balmanoukian A, Loriot Y. Atezolizumab in patients with locally advanced and metastatic urothelial carcinoma who have progressed following treatment with platinum-based chemotherapy: a single-arm, multicentre, phase 2 trial. Lancet. 2016;387(10031):1909-20.

51. Fehrenbacher $L$, Spira A, Ballinger $M$, Kowanetz $M$, Vansteenkiste J, Mazieres J, Park K, Smith D, Artal-Cortes A, Lewanski C. Atezolizumab versus docetaxel for patients with previously treated non-small-cell lung cancer (POPLAR): a multicentre, open-label, phase 2 randomised controlled trial. Lancet. 2016;387(10030):1837-46.

52. Rittmeyer A, Barlesi F, Waterkamp D, Park K, Ciardiello F, von Pawel J, Gadgeel SM, Hida T, Kowalski DM, Dols MC. Atezolizumab versus docetaxel in patients with previously treated non-small-cell lung cancer (OAK): a phase 3, openlabel, multicentre randomised controlled trial. Lancet. 2017;389(10066):255-65.

53. Postow MA, Chesney J, Pavlick AC, Robert C, Grossmann K, McDermott D, Linette GP, Meyer N, Giguere JK, Agarwala SS, et al. Nivolumab and Ipilimumab versus Ipilimumab in Untreated Melanoma. New England J Med. 2015;372(21):2006-17.

54. Wolchok JD, Kluger H, Callahan MK, Postow MA, Rizvi NA, Lesokhin AM, Segal NH, Ariyan CE, Gordon R-A, Reed K. Nivolumab plus ipilimumab in advanced melanoma. N Engl J Med. 2013;369(2):122-33.

55. Larkin J, Chiarion-Sileni V, Gonzalez R, Grob JJ, Cowey CL, Lao CD, Schadendorf D, Dummer R, Smylie M, Rutkowski P. Combined nivolumab and ipilimumab or monotherapy in untreated melanoma. N Engl J Med. 2015;2015(373):23-34.

56. Wolchok JD, Chiarion-Sileni V, Gonzalez R, Rutkowski P, Grob J-J, Cowey CL, Lao CD, Wagstaff J, Schadendorf D, Ferrucci PF. Overall survival with combined nivolumab and ipilimumab in advanced melanoma. N Engl J Med. 2017;377(14):1345-56.

57. Weber JS, Gibney G, Sullivan RJ, Sosman JA, Slingluff Jr CL, Lawrence DP, Logan TF, Schuchter LM, Nair S, Fecher L. Sequential administration of nivolumab and ipilimumab with a planned switch in patients with advanced melanoma (CheckMate 064): an open-label, randomised, phase 2 trial. Lancet Oncol. 2016;17(7):943-55.

58. Long GV, Atkinson V, Cebon JS, Jameson MB, Fitzharris BM, McNeil CM, Hill AG, Ribas A, Atkins MB, Thompson JA. Standard-dose pembrolizumab in combination with reduced-dose ipilimumab for patients with advanced melanoma (KEYNOTE029): an open-label, phase 1b trial. Lancet Oncol. 2017:18(9):1202-10.

59. Atkins MB, Choueiri TK, Hodi FS, Thompson JA, Hwu W-J, McDermott DF, Brookes M, Tosolini A, Ebbinghaus S, Yang Z. Pembrolizumab (MK-3475) plus low-dose ipilimumab (IPI) in patients (pts) with advanced melanoma (MEL) or renal cell carcinoma (RCC): data from the KEYNOTE-029 phase 1 study. J Clin Oncol. 2015;33(15_suppl):3009.

60. Hellmann M, Gettinger S, Goldman J, Brahmer J, Borghaei H, Chow L, Ready N, Gerber D, Juergens R, Shepherd F. CheckMate 012: safety and efficacy of first-line (1L) nivolumab (nivo; N) and ipilimumab (ipi; I) in advanced (adv) NSCLC. J Clin Oncol. 2016;34(Suppl):abstr 3001.

61. Gadgeel S, Stevenson J, Langer C, Gandhi L, Borghaei H, Patnaik A. Pembrolizumab (pembro) plus chemotherapy as front-line therapy for advanced NSCLC: KEYNOTE-021 cohorts AC. Proc Am Soc Clin Oncol. 2016;

62. Antonia S, Goldberg SB, Balmanoukian A, Chaft JE, Sanborn RE, Gupta A, Narwal R, Steele K, Gu Y, Karakunnel JJ. Safety and antitumour activity of durvalumab plus tremelimumab in non-small cell lung cancer: a multicentre, phase 1b study. Lancet Oncol. 2016;17(3):299-308. 
63. Doyle C. Combination immunotherapy superior to monotherapy in patients with melanoma. Am Health Drug Benefits. 2015;8(Spec Issue):41.

64. Topalian SL, Hodi FS, Brahmer JR, Gettinger SN, Smith DC, McDermott DF, Powderly JD, Carvajal RD, Sosman JA, Atkins MB. Safety, activity, and immune correlates of anti-PD-1 antibody in cancer. N Engl J Med. 2012;366(26):2443-54.

65. Larkin J, Chiarion-Sileni V, Gonzalez R, Grob JJ, Cowey CL, Lao CD, Schadendorf D, Dummer R, Smylie M, Rutkowski P, et al. Combined Nivolumab and Ipilimumab or monotherapy in untreated melanoma. N Engl J Med. 2015;373(1):23-34.

66. Schadendorf D, Wolchok JD, Hodi FS, Chiarion-Sileni V, Gonzalez R, Rutkowski P, Grob J-J, Cowey CL, Lao CD, Chesney J. Efficacy and safety outcomes in patients with advanced melanoma who discontinued treatment with nivolumab and ipilimumab because of adverse events: a pooled analysis of randomized phase II and III trials. J Clin Oncol. 2017;35(34):3807-14.

67. Antonia SJ, López-Martin JA, Bendell J, Ott PA, Taylor M, Eder JP, Jäger D, Pietanza MC, Le DT, de Braud F. Nivolumab alone and nivolumab plus ipilimumab in recurrent small-cell lung cancer (CheckMate 032): a multicentre, open-label, phase 1/2 trial. Lancet Oncol. 2016;17(7):883-95.

68. Basch E, Reeve BB, Mitchell SA, Clauser SB, Minasian LM, Dueck AC, Mendoza TR, Hay J, Atkinson TM, Abernethy AP. Development of the National Cancer Institute's patient-reported outcomes version of the common terminology criteria for adverse events (PRO-CTCAE). J Natl Cancer Inst. 2014;106(9):dju244.

69. Friedman CF, Clark V, Raikhel AV, Barz T, Shoushtari AN, Momtaz P, Callahan MK, Wolchok JD, Chapman PB, Hellmann MD. Thinking critically about classifying adverse events: incidence of pancreatitis in patients treated with nivolumab+ ipilimumab. JNCI. 2017;109(4):djw260.

70. Reck M, Rodríguez-Abreu D, Robinson AG, Hui R, Csőszi T, Fülöp A, Gottfried M, Peled N, Tafreshi A, Cuffe S. Pembrolizumab versus chemotherapy for PD-L1positive non-small-cell lung cancer. N Engl J Med. 2016;375(19):1823-33.

71. Top-Line B-MSA: Results from CheckMate- 026, a Phase 3 Study of Opdivo (nivolumab) in Treatment-Naiive Patients with Advanced Non-Small Cell Lung Cancer. Dostupné na:<. 2016. p. 8.https://news.bms.com/press-release/bristolmyers/ bristol-myers-squibb-announces-top-line-resultscheckmate-026-phase-3-stu.

72. Socinski M, Creelan B, Horn L, Reck M, Paz-Ares L, Steins M, Felip E, van den Heuvel M, Ciuleanu T, Badin F. NSCLC, metastaticCheckMate 026: a phase 3 trial of nivolumab vs investigator's choice (IC) of platinum-based doublet chemotherapy (PT-DC) as first-line therapy for stage iv/recurrent programmed death ligand 1 (PD-L1) - positive NSCLC. Ann Oncol. 2016; 27(suppl 6):LBA7_PR.

73. Muro K, Chung HC, Shankaran V, Geva R, Catenacci D, Gupta S, Eder JP, Golan T, Le DT, Burtness B. Pembrolizumab for patients with PD-L1-positive advanced gastric cancer (KEYNOTE-012): a multicentre, open-label, phase 1b trial. Lancet Oncol. 2016;17(6):717-26.

74. Le DT, Yoshino T, Jäger D, Andre T, Bendell JC, Wang R, Kang SP, Koshiji M, Diaz LA. KEYNOTE-164: phase II study of pembrolizumab (MK-3475) for patients with previously treated, microsatellite instability-high advanced colorectal carcinoma. Proc Am Soc Clin Oncol. 2016

75. O'Neil BH, Wallmark JM, Lorente D, Elez E, Raimbourg J, Gomez-Roca C, Ejadi S, Piha-Paul SA, Stein MN, Razak ARA. Safety and antitumor activity of the anti-PD-1 antibody pembrolizumab in patients with advanced colorectal carcinoma. PLoS One. 2017:12(12):e0189848.

76. Diaz LA, Marabelle A, Delord J-P, Shapira-Frommer R, Geva R, Peled N, Kim TW, Andre T, Van Cutsem E, Guimbaud R. Pembrolizumab therapy for microsatellite instability high (MSI-H) colorectal cancer (CRC) and non-CRC. Proc Am Soc Clin Oncol. 2017;

77. Zimmer L, Apuri S, Eroglu Z, Kottschade LA, Forschner A, Gutzmer R, Schlaak $M$, Heinzerling L, Krackhardt AM, Loquai C. Ipilimumab alone or in combination with nivolumab after progression on anti-PD-1 therapy in advanced melanoma. Eur J Cancer. 2017;75:47-55.

78. Prithviraj P, McArthur GA, Atkinson V, Parente P, Andrews MC, Parakh S, Cebon JS, Klein O. Efficacy and toxicity of treatment with the anti-CTLA-4 antibody Ipilimumab in patients with metastatic melanoma who have progressed on anti-PD-1 therapy. Proc Am Soc Clin Oncol. 2015;33(15_ suppl):9059.

79. Steuer C, Papadimitrakopoulou V, Herbst R, Redman M, Hirsch F, Mack P, Ramalingam S, Gandara D. Innovative clinical trials: the LUNG-MAP study. Clin Pharmacol Therapeutics. 2015;97(5):488-91.

80. Taylor M, Antonia S, Bendell J, Calvo E, Jäger D, de Braud F, Ott PA, Pietanza MC, Horn L, Le DT: Phase I/II study of nivolumab with or without ipilimumab for treatment of recurrent small cell lung cancer (SCLC): CA209032. J Immunotherapy Cancer 2015, 3(2):P376.

\section{Ready to submit your research? Choose BMC and benefit from:}

- fast, convenient online submission

- thorough peer review by experienced researchers in your field

- rapid publication on acceptance

- support for research data, including large and complex data types

- gold Open Access which fosters wider collaboration and increased citations

- maximum visibility for your research: over $100 \mathrm{M}$ website views per year

At BMC, research is always in progress.

Learn more biomedcentral.com/submissions 